\title{
Interpolação Hermiteana usando Bases de Funções Radiais e Funções Regradas
}

\author{
Regina Célia Guapo Pasquini
}

Orientador: Prof. Dr. Valdir Antonio Menegatto

Dissertação apresentada ao Instituto de Ciências Matemáticas de São Carlos-USP, como parte dos requisitos para obtenção do título de "Mestre em Ciências - Área: Matemática”.

\author{
USP - São Carlos
}

1997 
Ao meu amado esposo José Almir Ao meu "pequeno" João Felipe 


\section{Agradecimentos ${ }^{1}$}

À Deus pelo dom da vida.

Ao Prof. Dr. Valdir Antonio Menegatto pela paciência e amizade que mesmo quando a distância, soube me orientar de forma dedicada e competente.

Aos meus pais Janira e Deorande, pelo apoio e incentivo constante durante toda a minha vida escolar.

Ao meu esposo e companheiro de sempre, José Almir, pelo carinho, compreensão e conforto nos momentos difíceis, sabendo suportar minha ausência e tendo forças por nós dois para prosseguir.

Ao meu filho João Felipe, que trouxe tanto amor e completividade para nossas vidas.

À minha avó Darcília pelas orações.

À toda minha família.

Aos meus professores de graduação e especialização do DMA, UEM.

À grande amiga Rosali pelo exemplo de otimismo, e à Kelen pela amizade dedicada.

Aos colegas de turma, Ana, Marcio, Simone e Toninho pelo apoio e compreensão dados durante o nosso convívio.

Aos meus professores do mestrado, Prof. Dr. Alexandre Nolasco de Carvalho, Profa. Dra. Ires Dias, Prof. Dr. Luis Augusto da Costa Ladeira, Prof. Dra. Maria Aparecida Soares Ruas, Prof. Dr. Oziride Manzoli Neto, Prof. Dr. Valdir Antonio Menegatto, Prof. Dr. Sergio Luis Zani por me formar e informar.

\footnotetext{
${ }^{1}$ Este trabalho teve suporte financeiro do CNPq.
} 
À Coordenação da área de Matemática, na pessoa do Prof. Dr. Alexandre, e ao Prof. Dr. Valdir, que permitiram que eu pudesse ficar junto do meu filho quando ele mais precisou de mim.

À Ana Paula pela colaboração nos estudos e correções.

Aos colegas de viagem, Valdeni, Alexandra, Adriana, Marcos e companhia, pelas horas de bate papo tornando assim a distância menos longa.

Às secretárias Laura, Beth e Marília pela simpatia, atenção e eficiência com que sempre me atenderam.

Aos novos colegas de São Carlos que me proporcionaram momentos alegres.

Ao Marcelo pela paciência em me ouvir nas horas em que precisei.

À todos que direta, ou indiretamente, cooperaram para a realização deste trabalho. 


\section{Deus, pastor dos homens}

O Senhor é meu pastor, nada me faltará.

Em verdes prados ele me faz repousar.

Conduz-me junto às águas refrescantes,

Restaura as forças de minha alma.

Pelos caminhos retos ele me leva,

Por amor do seu nome.

Ainda que eu atravesse o vale escuro,

Nada temerei, pois estais comigo.

Vosso bordão e vosso báculo

São o meu amparo

Preparais para mim a mesa

A vista de meus inimigos.

Derramais o perfume sobre minha cabeça,

Transborda a minha taça.

A vossa bondade e misericórdia hão de seguir-me

Por todos os dias da minha vida.

E habitarei na casa do senhor

Por longos dias.

(Salmo 22) 


\begin{abstract}
We study multivariable scattered Hermite interpolation via two distinct methods.

The first one is an extension of the so-called radial-basis function method. In this case, the solutions are constructed from the Fourier transforms of certain finite measures and the associated interpolation matrices are always positive definite.

The second one is an extension of the method known as interpolation by ridge functions. The existence of solutions by this method depends upon the positive definiteness of a certain matrix, here called the characteristic matrix of the problem.

Certain classes of completely monotone functions are used to achieve simple realizations of the methods.
\end{abstract}




\section{Resumo}

Estudamos problemas de interpolação hermitiana em várias variáveis através de dois métodos distintos.

O primeiro é uma extensão do método das bases de funções radiais para interpolação em várias variáveis. Ele baseia-se na transformada de Fourier de uma medida previamente fixada. As matrizes de interpolação produzidas por esse método são sempre positivas definidas.

O segundo é uma extensão do método das funções regradas. O problema tem solução por esse método se, e somente se, uma certa matriz, denominada de matriz característica do problema, for positiva definida.

Certas classes de funções completamente monótonas são utilizadas na construção de implementações dos métodos estudados. 


\section{Índice}

1 Introdução 1

2 Interpolação Hermiteana Usando a Transformada de Fourier $\quad 6$

$\begin{array}{llr}3 & \text { A Matriz Característica } & 15\end{array}$

4 Algumas Classes de Funções Completamente Monótonas 25

5 Soluções do Problema 1 Via Funções Completamente Monótonas 33

6 Soluções do Problema 2 Via Funções Completamente Monótonas 39

$\begin{array}{ll}\text { Bibliografia } & 47\end{array}$ 


\section{Capítulo 1}

\section{Introdução}

Sejam $\mathcal{N}:=\left\{x_{1}, x_{2}, \ldots, x_{n}\right\}$ e $X$ subconjuntos de $\mathbb{R}^{d}$ e $b_{1}, b_{2}, \ldots, b_{n}$ escalares quaisquer. Nesse trabalho, o termo interpolação refere-se ao seguinte problema: encontrar uma função $f: X \rightarrow \mathbb{C}$, de algum tipo pré-determinado, de tal forma que

$$
f\left(x_{i}\right)=b_{i}, \quad 1 \leq i \leq n,
$$

Se uma tal função $f$ existe, ela é chamada função interpoladora do problema. As condições (1.1) são chamadas condições de interpolação do problema.

Dentre os métodos empregados na literatura para resolver o problema acima, destacamos dois deles. O primeiro, conhecido como o método das bases de funções radiais, consiste em buscar as soluções em um espaço gerado por funções da forma

$$
\phi\left(\left\|x-x_{1}\right\|\right), \phi\left(\left\|x-x_{2}\right\|\right), \ldots, \phi\left(\left\|x-x_{n}\right\|\right),
$$

onde $\|\cdot\|$ é a norma usual de $\mathbb{R}^{d}$ e $\phi: \mathbb{R} \longrightarrow \mathbb{C}$ é uma função qualquer sujeita à nossa escolha. Note que cada função na lista acima é uma translação da função radial $\phi(\|\cdot\|)$. Escrerendo-se então a solução procurada na forma

$$
f(. r)=\sum_{j=1}^{n} c_{j} \phi\left(\left\|x-x_{j}\right\|\right), \quad c_{j} \in \mathbb{C}, \quad 1 \leq j \leq n,
$$


e impondo-se as condições de interpolação, obtemos o sistema

$$
\sum_{j=1}^{n} c_{j} \phi\left(\|\left(x_{i}-x_{j} \|\right)=b_{i}, \quad 1 \leq i \leq n .\right.
$$

A determinação de $f$ reduz-se portanto à resolução desse sistema cujas incógnitas são $c_{1}, c_{2}, \ldots, c_{n}$. A matriz do mesmo, comumente chamada de matriz de interpolação do problema, é a matriz $A$ com entradas

$$
A_{i j}=\phi\left(\|\left(x_{i}-x_{j} \|\right), \quad 1 \leq i, j \leq n .\right.
$$

O problema de interpolação tem solução única quaisquer que sejam os $b_{i}$ 's se, e somente se, a matriz $A$ acima for não singular. Portanto a escolha da função $\phi$ que deve gerar a solução do problema é de extrema importância.

O outro método, emprega combinações lineares do que nós denominamos funções regradas. Uma função $g: \mathbb{R}^{d} \rightarrow \mathbb{R}$ é dita ser uma função regrada se existir uma função $h: \mathbb{R} \rightarrow \mathbb{R}$ e um vetor $a \in \mathbb{R}^{d}$ tal que

$$
g(x)=h(\langle a, x\rangle), \quad x \in \mathbb{R}^{d},
$$

onde $\langle\cdot, \cdot\rangle$ denota o produto interno usual em $\mathbb{R}^{d}$. O vetor a é chamado de direção da função $g$. Para uma direção a fixada, denotaremos por $\mathcal{R}_{a}$ o espaço formado por todas as funções regradas com direção $a$. Dado um conjunto $\mathcal{T}=\left\{a_{1}, a_{2}, \ldots, a_{\omega}\right\}$ de direções duas a duas linearmente independentes, denotaremos por $\mathcal{R}_{\mathcal{T}}$ a soma dos espaços $\mathcal{R}_{a_{1}}, \mathcal{R}_{a_{2}}, \ldots, \mathcal{R}_{a_{\omega}}$. Em símbolos,

$$
\mathcal{R}_{\mathcal{T}}:=\sum_{m=1}^{\omega} \mathcal{R}_{a_{m}}
$$

O método propriamente dito consiste em buscar funções $f$ em $\mathcal{R}_{\mathcal{T}}$ tais que $f\left(x_{i}\right)=b_{i}$, $1 \leq i \leq n$. Este método não é tão efetivo quanto o anterior. É sabido, por exemplo 
([2]), que para qualquer conjunto $\mathcal{T}$, sempre existem um conjunto $\mathcal{N}$ e escalares $b_{1}, b_{2}, \ldots, b_{n}$, para os quais o problema descrito não tem solução. Os dois métodos descritos, incluindo-se aqui a determinação de soluções bem como outras questões atreladas a esta, têm sido e ainda estão sendo estudadas. Neste trabalho estudaremos a resolubilidade e algumas implementações de algumas generalizações dos problemas acima para o âmbito de interpolação hermiteana. Portanto, não é objetivo do mesmo, discutir uma possível comparação entre os métodos nem a efetividade de cada um deles. O termo interpolação hermiteana refere-se a problemas de interpolação onde as condições de interpolação acima descritas são substituídas por condições envolvendo certas derivadas da função $f$. Obviamente, certas condições tem que ser impostas desde o começo para garantir a existência das derivadas da função $f$ envolvidas no processo.

Passemos então à descrição dos problemas de interpolação hermiteana que estudaremos. Além do conjunto $\mathcal{N}$, suponha que um conjunto $\mathcal{P}:=\left\{p_{1}, p_{2}, \ldots, p_{r}\right\}$ de polinômios homogêneos em $d$ variáveis, com coeficientes complexos, seja dado. Sejam ainda $p_{\mu}(D), \mu=1,2, \ldots, r$, os operadores diferenciais associados.

Problema 1. Assuma que $\mathcal{P}$ seja linearmente independente. Sejam $h_{1}, h_{2}, \ldots, h_{r}$ : $\mathbb{R}^{d} \rightarrow \mathbb{C}$ funções contínuas, de tal modo que $p_{\mu}(D) h_{\nu}, \mu, \nu=1,2, \ldots, r$, também sejam contínuas, $e>: \mathcal{N} \rightarrow \mathbb{C}^{r}$ uma função qualquer. Encontrar uma função $f$ no espaço gerado pelas $n r$ funções $h_{\nu}\left(x-x_{j}\right), \nu=1,2, \ldots, r, j=1,2, \ldots, n$, de tal modo que a função $F: \mathbb{R}^{d} \rightarrow \mathbb{C}^{r}$ definida por

$$
F:=\left(p_{1}(D) f, p_{2}(D) f, \ldots, p_{r}(D) f\right)
$$

satisfaça $F\left(x_{j}\right)=\perp\left(x_{j}\right), j=1,2 \ldots, n$. 
Quando as condições de interpolação são impostas, ou seja, quando fazemos

$$
F\left(x_{j}\right)=\Delta\left(x_{j}\right), \quad 1 \leq j \leq n,
$$

obtemos

$$
p_{\mu}(D) f\left(x_{j}\right)=\Delta\left(x_{j}\right), \quad 1 \leq j \leq n, \quad 1 \leq \mu \leq r .
$$

Escrevendo-se agora

$$
f(x)=\sum_{\nu=1}^{r} \sum_{k=1}^{n} c_{\nu k} h_{\nu}\left(x-x_{k}\right),
$$

com os $c_{\nu k}$ 's em $\mathbb{C}$, e $\Delta\left(x_{j}\right)=\left(b_{1 j}, b_{2 j}, \ldots, b_{r j}\right), 1 \leq j \leq n$, o sistema (1.2) fica na forma

$$
\sum_{\nu=1}^{r} \sum_{k=1}^{n} c_{\nu k} p_{\mu}(D) h_{\nu}\left(x_{j}-x_{k}\right)=b_{\mu j}, \quad 1 \leq \mu \leq r, \quad 1 \leq j \leq n .
$$

A matriz desse sistema é uma matriz $A$ hermiteana de ordem $n r \times n r$, dada em blocos por $A=\left(A_{\mu \nu}\right)_{\mu, \nu=1}^{r}$, onde

$$
A_{\mu \nu}=\left(p_{\mu}(D) h_{\nu}\left(x_{j}-x_{k}\right)\right)_{j, k=1}^{n}
$$

O Problema 1 tem solução única qualquer que seja a função $\Delta$ se, e somente se, a matriz $A$ for não singular. Note que, em princípio, esse problema não envolve funções radiais diretamente, mas as implementações do problema serão feitas com funções radiais somente. No Capítulo II, discutiremos a resolubilidade do problema acima para uma escolha geral das funções $h_{\nu}$; na verdade todas serão definidas a partir de uma única função $g$ pertencente a uma classe relativamente grande de funções. Tal classe inclui muitas funções radiais sabidamente importantes dentro do contexto de interpolação em várias variáveis. As matrizes $A$ nesse caso, serão sempre positivas definidas e portanto, seus autoralores serão todos positivos. No Capítulo V explicitaremos várias classes de funções radiais que encaixam-se nesta teoria. Extrapolando 
um pouco os resultados do Capítulo $V$, exibiremos uma classe de funções para as quais as matrizes $A$ tem quase todos os autovalores positivos, havendo no máximo uma possível excessão. Concluindo nossa discussão do Problema 1, nós observamos que o mesmo reduz-se àquele discutido no início deste capítulo, quando $\mathcal{P}$ é composto somente pelo polinômio constante igual a $1, h_{1}=h_{2}=\cdots=h_{r}$ e $h_{1}$ é radial.

Para o problema restante, além de $\mathcal{N}, \mathcal{P}$, e $\Delta$ considere os conjuntos $\mathcal{T}$ e $\mathcal{R}_{\mathcal{T}}$ anteriormente descritos. Aqui, podemos assumir que todos os polinômios tem coeficientes reais.

Problema 2. Encontrar uma função $f$ em $\mathcal{R}_{\mathcal{T}}$ tal que

$$
\left[p_{\mu}(D) f\right]\left(x_{j}\right)=\Delta\left(x_{j}\right), \quad 1 \leq j \leq n, \quad 1 \leq \mu \leq r .
$$

Uma discussão completa desse problema é feita no Capítulo III, onde nós mostraremos que o problema acima tem solução qualquer que seja a função $\Delta$ se, e somente se, uma certa matriz, dependente de $\mathcal{P}$ e $\mathcal{T}$ somente, for não singular. No Capítulo VI, nós mostramos duas implementações do problema, utilizando as mesmas classes de funções usadas na implementação do Problema 1. Finalmente, observe que o Problema 2 reduz-se ao segundo problema discutído no começo do capítulo quando $\mathcal{P}$ é unitário e formado pelo polinômio constante igual a 1.

As referências básicas para esse trabalho são: [5], [7] e [8]. Várias outras secundárias, mas não menos importantes, serão creditadas no momento oportuno. 


\section{Capítulo 2}

\section{Interpolação Hermiteana Usando a Transformada de Fourier}

Neste Capítulo, estudaremos exclusivamente o Problema 1 descrito no capítulo anterior. Precisamente, mostraremos que uma classe relativamente grande de funções (não necessariamente radiais) podem ser utilizadas para a construção de soluções do problema.

Antes de enunciarmos o resultado principal desse capítulo, provaremos vários resultados preliminares. Os dois primeiros, referem-se à independência linear de um certo conjunto de funções polinômio-exponenciais.

Lema 2.1 Sejam $l_{1}, l_{2}, \ldots, l_{s}$ inteiros positivos e $r_{1}, r_{2}, \ldots, r_{s}$ números reais distintos. Então, o conjunto $\cup_{j=1}^{s}\left\{t^{k} \exp \left(r_{j} t\right): 0 \leq k \leq l_{j}-1\right\}$ é linearmente independente sobre $\mathbb{C}$, em qualquer intervalo da reta.

Prova Assumamos as hipóteses e suponhamos que o conjunto do lema seja linearmente dependente em algum interralo $I$ de $\mathbb{R}$. Então, existem números complexos 
$c_{j k}, 0 \leq k \leq l_{j}-1,1 \leq j \leq s$, não todos nulos, tais que

$$
\sum_{j=1}^{s} \sum_{k=0}^{l_{j}-1} c_{j k} t^{k} e^{r_{j} t}=0, \quad t \in I
$$

ou, equivalentemente,

$$
\sum_{j=1}^{s}\left(\sum_{k=0}^{l_{j}-1} c_{j k} t^{k}\right) e^{r_{j} t}=0, \quad t \in I .
$$

Definindo-se

$$
p_{j}(t):=\sum_{k=0}^{l_{j}-1} c_{j k} t^{k}, \quad 1 \leq j \leq s,
$$

podemos então escrever a última igualdade na forma

$$
\sum_{j=1}^{s} p_{j}(t) e^{r_{j} t}=0, \quad t \in I
$$

Como pelo menos um dos coeficientes $c_{j k}$ é não nulo, pelo menos um dos polinômios $p_{j}$ é não identicamente nulo. Sem perda de generalidade, assumamos que todos os $p_{j}$ sejam não nulos. Em particular, $p_{s}$ não é identicamente nulo em $I$. Dividindo (2.1) por $\exp \left(r_{1} t\right)$ obtemos

$$
p_{1}(t)+\sum_{j=2}^{s} p_{j}(t) e^{\left(r_{j}-r_{1}\right) t}=0, \quad t \in I .
$$

Diferenciando-se a relação anterior até que o termo polinomial desapareça, chegamos a uma expressão da forma

$$
\sum_{j=2}^{s} q_{j}(t) e^{\left(r_{j}-r_{1}\right) t}=0, \quad t \in I,
$$

onde cada $q_{j}, j=2, \ldots, s$, tem o mesmo grau que $p_{j}$. Em particular, $q_{s}$ não é identicamente nulo. Repetindo-se esse processo um número finito de vezes, finalmente obtemos uma expressão da forma

$$
\bar{q}_{s}(t) e^{\left(r_{s}-r_{s-1}\right) t}=0, \quad t \in I .
$$


onde o polinômio $\bar{q}_{s}$ é um polinômio não identicamente nulo. Como a exponencial nunca se anula, isto é uma óbvia contradição.

Nós usaremos a seguinte extensão do lema acima.

Corolário 2.1 Assuma as hipóteses do lema anterior. Então o conjunto de funções $\cup_{j=1}^{s}\left\{t^{k} \exp \left(i r_{j} t\right): 1 \leq k \leq l_{j}-1\right\}$ é linearmente independente sobre $\mathbb{C}$, em qualquer intervalo da reta.

Prova Basta imitar a prova do lema anterior.

O próximo lema é de caráter geométrico.

Lema 2.2 Se $x_{1}, x_{2}, \ldots, x_{n}$ são vetores distintos em $\mathbb{R}^{d}$, então existe um vetor y em $\mathbb{R}^{d}$ tal que $\left\langle y, x_{i}\right\rangle \neq\left\langle y, x_{j}\right\rangle, i \neq j$. Além disso, o conjunto formado por todos os tais y é aberto em $\mathbb{R}^{d}$.

Prova Primeiramente, note que para $i$ e $j$ fixos, cada conjunto formado pelos $x$ de $\mathbb{R}^{d}$ que satisfazem $\left\langle x, x_{i}\right\rangle=\left\langle x, x_{j}\right\rangle$, é um hiperplano de $\mathbb{R}^{d}$, isto é, um subespaço de $\mathbb{R}^{d}$ de dimensão $d-1$. Portanto, a primeira parte do enunciado do lema corresponde a dizer que dados uma quantidade finita de hiperplanos de $\mathbb{R}^{d}$, é sempre possível encontrar um y que não está em nenhum deles. Como uma quantidade finita de hiperplanos não pode prencher todo o $\mathbb{R}^{d}$, a primeira parte do lema segue.

Quanto à segunda parte, basta notar que a o complemento de um hiperplano é um aberto de $\mathbb{R}^{d}$. Logo, essa parte segue do fato de que uma intersecção finita de abertos de $\mathbb{R}^{d}$ é ainda um aberto de $\mathbb{R}^{d}$.

Podemos agora enunciar o resultado técnico principal desse capítulo. 
Teorema 2.1 Sejam $x_{1}, x_{2}, \ldots, x_{n}$ pontos distintos de $\mathbb{R}^{d}$ e $\mathcal{P}$ como no Problema 1. Se $c_{11}, c_{12}, \ldots, c_{1 n}, c_{21}, c_{22}, \ldots, c_{2 n}, \ldots, c_{r 1}, c_{r 2}, \ldots, c_{n r}$ são números complexos, não todos nulos, então a função $\varphi: \mathbb{R}^{d} \rightarrow \mathbb{C}$ dada por

$$
\varphi(x)=\sum_{\mu=1}^{r} \sum_{j=1}^{n} c_{\mu j} p_{\mu}(i x) e^{i\left\langle x, x_{j}\right\rangle}
$$

não é identicamente nula.

Prova Assuma as hipóteses e suponha que $\varphi$ seja identicamente nula. Seja $H$ o conjunto descrito na segunda afirmação do lema anterior. Para y em $H$ e $t>0$, temos então que

$$
\sum_{\mu=1}^{r} \sum_{j=1}^{n} c_{\mu j} p_{\mu}(i t y) e^{i t\left\langle y, x_{j}\right\rangle}=0
$$

Usando o fato dos polinômios serem homogêneos e reagrupando convenientemente as somas, a igualdade acima reduz-se a

$$
\sum_{j=1}^{n}\left(\sum_{\mu=1}^{r} p_{\mu}(i y) c_{\mu j}\right) t^{\alpha_{\mu}} e^{i\left\langle y, x_{j}\right\rangle t}=0, \quad y \in H
$$

onde $\alpha_{\mu}$ é o grau de $p_{\mu}$. Pelo Corolário 2.1, todos os coeficientes na igualdade acima têm que ser nulos. Em outras palavras,

$$
\sum_{\mu=1}^{r} p_{\mu}(i y) c_{\mu j}=0, \quad 1 \leq j \leq n, \quad y \in H
$$

Pelo lema anterior, sabemos que $H$ é aberto em $\mathbb{R}^{d}$. Portanto, a última igualdade acima vale para todo $y \mathrm{em} \mathbb{R}^{d}$. Como os polinômios são homogêneos, podemos então escrever tal igualdade na forma

$$
\sum_{\mu=1}^{r} d_{\mu j} p_{\mu}(x)=0 . \quad x \in \mathbb{R}^{d},
$$

onde cada $d_{\mu j}$ é uma certa potência de $i$ vezes $c_{\mu j}$. Como os polinômios $p_{\mu}$ formam um conjunto linearmente independente, necessariamente temos que $d_{\mu j}=0$ para todo $\mu$ e todo $j$. Em particular. $c_{\mu j}=0$, para todo $\mu$ e todo $j$. 
A seguir, introduziremos a classe de funções que utilizaremos para a construção de soluções do Problema 1.

Seja $\beta$ uma medida de Borel finita em $\mathbb{R}^{d}$. A transformada de Fourier $\widehat{\beta}$ de $\beta$ é a função dada por

$$
\widehat{\beta}(x)=\int_{\mathbb{R}^{d}} e^{i\langle x, \xi\rangle} d \beta(\xi) .
$$

Seja $\mathcal{P}$ como no capítulo anterior. Neste capítulo, denotaremos por $M_{\mathcal{P}}$ o conjunto de todas as medidas $\beta$ de Borel, finitas e positivas em $\mathbb{R}^{d}$, que não são concentradas em um subconjunto de $\mathbb{R}^{d}$ com medida de Lebesgue nula, e que satisfazem a seguinte condição:

$$
\int_{\mathbb{R}^{d}}\|\xi\|^{2 \gamma} d \beta(\xi)<\infty, \quad \gamma=\max \left\{\alpha_{\nu}: 1 \leq \nu \leq r\right\}
$$

Na equação acima, bem como no restante do trabalho, $\alpha_{\mu}$ denota o grau de $p_{\mu}$ em $\mathcal{P}$. Em analogia com a nossa notação para a transformada de Fourier de uma medida escreveremos $\widehat{M}_{\mathcal{P}}$ para denotar o conjunto de todas as transformadas de Fourier de medidas de $M_{\mathcal{P}}$.

Antes de enunciarmos as propriedades de $\widehat{M}_{\mathcal{P}}$ que serão utilizadas nesse capítulo, necessitamos registrar a seguinte versão do teorema da diferenciação sob o sinal de integração. Ela será usada em pelo menos duas ocasiões distintas neste trabalho. Uma prova detalhada dessa versão pode ser encontrada ná página 154 em [4]. As componentes de um vetor $x$ qualquer de $\mathbb{R}^{d}$ serão denotadas por $x^{1}, x^{2}, \ldots, x^{d}$, respectivamente.

Lema 2.3 Sejam $(X, \beta)$ um espaço de medida, $U$ um aberto de $\mathbb{R}^{d}$ e $\phi: U \times X \rightarrow \mathbb{C}$ uma função satisfazendo as seguintes hipóteses:

i) Para cada y de $U$, a função $\phi(y, \cdot)$ é integrável em relação a $\beta$; 
ii) Para todo $\xi$ em $X$ (a menos de um conjunto de medida nula em relação a $\beta$ ), a derivada parcial de $\phi(\cdot, \xi)$ em relação a $y^{j}$ existe em todos os pontos de $U$;

iii) Existe uma função $h: X \rightarrow \mathbb{C}$, integrável em relação a $\beta$, tal que $\left|\left(\partial \phi / \partial y^{j}\right)(y, \xi)\right|$ $\leq h(\xi)$, for all $(y, \xi)$ em $U \times X$.

Então, a função

$$
\Phi(y):=\int_{X} \phi(y, \xi) d \beta(\xi), \quad y \in U
$$

possui derivada parcial em relação a $y^{j}$ em $U$ e

$$
\frac{\partial \Phi}{\partial y^{j}}(y)=\int_{\mathbb{R}^{d}} \frac{\partial \phi}{\partial y^{j}}(y, \xi) d \beta(\xi) .
$$

De agora em diante, se $p$ é um polinômio em $d$ variáveis escreveremos $\bar{p}$ para denotar o polinômio obtido de $p$ via conjugação de seus coeficientes.

Lema 2.4 Se g é um elemento de $\widehat{M}_{\mathcal{P}}$ e p é um polinômio qualquer, então

$$
p_{\mu}(D) \overline{p_{\nu}}(D) g(x)=\int_{\mathbb{R}^{d}} p_{\mu}(i \xi) \overline{p_{\nu}}(i \xi) e^{i\langle x, \xi\rangle} d \beta(\xi), \quad x \in \mathbb{R}^{d}, \quad 1 \leq \mu, \nu \leq r .
$$

Prova Basta escrever $g$ na forma $g=\widehat{\beta}$, para alguma medida de Borel finita e positiva $\beta$ sobre $\mathbb{R}^{d}$ e então aplicar o lema anterior um número finito de vezes.

Finalmente podemos enunciar e provar o teorema principal desse capítulo.

Teorema 2.2 Sejam $\mathcal{P}$ como no Problema 1, g uma funçăo de $\widehat{M}_{\mathcal{P}}$ e defina $h_{\nu}=$ $(-1)^{\alpha_{\nu}} \overline{p_{\nu}}(D) g$. Então. a matriz de interpolação A correspondente ao Problema 1 é positiva definida. 
Prova Seja $\beta$ uma medida de Borel finita e positiva sobre $\mathbb{R}^{d}$ tal que $g=\widehat{\beta}$. A matriz $A$ do enunciado é dada em blocos por $A=\left(A_{\mu \nu}\right)_{\mu, \nu=1}^{r}$, onde cada $A_{\mu \nu}$ é uma matriz de ordem $n$ com entrada $j k$ dada por

$$
(-1)^{\alpha_{\nu}} p_{\mu}(D) \overline{p_{\nu}}(D) g\left(x_{j}-x_{k}\right) .
$$

Para mostrar que $A$ é positiva definida, mostraremos que para todo $c \in \mathbb{C}^{n r}$, vale $\bar{c}^{t} A c \geq 0$, com igualdade somente quando $c=0$. Note que $\bar{c}$ denota o vetor conjugado de $c$. Seja então $c \in \mathbb{C}^{n r}$ e escrevamos

$$
c=\left(c_{11}, c_{12}, \ldots, c_{1 n}, c_{21}, c_{22}, \ldots, c_{2 n}, \ldots, c_{r 1}, c_{r 2}, \ldots, c_{n r}\right) .
$$

Temos que

$$
\begin{aligned}
& \bar{c}^{t} A c=\sum_{j=1}^{n} \sum_{\nu=1}^{r} \sum_{k=1}^{n}(-1)^{\alpha_{\nu}} p_{\mu}(D) \overline{p_{\nu}}(D) g\left(x_{j}-x_{k}\right) \overline{c_{\mu j}} c_{\nu k} \\
& =\sum_{\mu=1}^{r} \sum_{j=1}^{m} \sum_{\nu=1}^{r} \sum_{k=1}^{n} \overline{c_{\mu j}} c_{\nu k} \int_{\mathbb{R}^{d}}(-1)^{\alpha_{\nu}} p_{\mu}(i \xi) \overline{p_{\nu}}(i \xi) e^{i\left\langle\xi, x_{j}-x_{k}\right\rangle} d \beta(\xi) \\
& =\int_{\mathbb{R}^{d}} \sum_{\mu=1}^{r} \sum_{\nu=1}^{r}(-1)^{\alpha_{\nu}} p_{\mu}(i \xi) \overline{p_{\nu}}(i \xi) \sum_{j=1}^{n} \sum_{k=1}^{n} \overline{c_{\mu j}} c_{\nu k} e^{i\left\langle\xi, x_{j}-x_{k}\right\rangle} d \beta(\xi) \\
& =\int_{\mathbb{R}^{d}} \sum_{\mu=1}^{r} \sum_{\nu=1}^{r}(-1)^{\alpha_{\nu}} p_{\mu}(i \xi) \overline{p_{\nu}}(i \xi)\left(\sum_{j=1}^{n} \overline{c_{\mu j}} e^{i\left\langle\xi, x_{j}\right\rangle}\right)\left(\overline{\left.\sum_{j=1}^{n} \overline{c_{\mu j}} e^{i\left\langle\xi, x_{j}\right\rangle}\right)}\right) d \beta(\xi) \\
& =\int_{\mathbb{R}^{d}}\left|\sum_{\mu=1}^{r} p_{\mu}(i \xi) \sum_{j=1}^{n} \overline{c_{\mu j}} e^{i\left\langle\xi, x_{j}\right\rangle}\right|^{2} d \beta(\xi) .
\end{aligned}
$$

A última expressão é obviamente não negativa. Portanto, resta mostramos que ela anula-se somente quando $c=0$. Assumamos então que $c \neq 0$. Pelas nossas hipóteses e pelo Teorema 2.1, a função

$$
\varphi(\xi):=\sum_{\mu=1}^{r} p_{\mu}(i \xi) \sum_{j=1}^{n} \overline{c_{\mu j}} e^{i\left\langle\xi, x_{j}\right\rangle}
$$


não é identicamente nula. Como $\varphi$ é analítica, o conjunto formado por todos os seus zeros tem medida nula. Como $\beta$ não está concentrada em um conjunto de medida nula de $\mathbb{R}^{d}$ então, neste caso,

$$
\int_{\mathbb{R}^{d}}|\varphi(\xi)|^{2} d \beta(\xi)>0,
$$

completando a prova do teorema.

Como dissemos anteriormente, exemplos mais concretos de realizações do teorema anterior serão estudados no Capítulo V. Automaticamente, exemplos de funções da classe $\widehat{M}_{\mathcal{P}}$ tem que estar à mão. Utilizaremos o restante deste capítulo para apresentar um tal exemplo. Na verdade, todos os demais exemplos neste trabalho dependerão deste.

Teorema 2.3 Para todo $\rho>0$, a função $G_{\rho}: \mathbb{R}^{d} \rightarrow \mathbb{R}$ dada pela expressão $G_{\rho}(x)=$ $\exp \left(-\rho\|x\|^{2}\right)$ está em $\widehat{M}_{\mathcal{P}}$.

Prova É suficiente observar que a seguinte fórmula clássica ([1]) vale:

$$
e^{-\rho x^{2}}=\frac{1}{2 \sqrt{\pi}} \int_{\mathbb{R}} e^{i \sqrt{\rho} x \xi} e^{-\xi^{2} / 4} d \xi, \quad x \in \mathbb{R} .
$$

Para obter uma fórmula análoga em $\mathbb{R}^{d}$, basta substituir $x$ por $x^{j}, 1 \leq j \leq d$, e multiplicar as integrais resultantes. O resultado é a fórmula

$$
\begin{aligned}
e^{-\rho\|\cdot x\|^{2}} & =\frac{1}{(4 \pi)^{d / 2}} \int_{\mathbb{R}^{d}} e^{i \sqrt{\rho}\langle x, \xi\rangle} e^{-\|\xi\|^{2} / 4} d \xi \\
& =\frac{1}{(4 \pi \rho)^{d / 2}} \int_{\mathbb{R}^{d}} e^{i\langle x, \xi\rangle} e^{-\|\xi\|^{2} / 4 \rho} d \xi .
\end{aligned}
$$

Note que aqui, $d \xi=d \xi^{1} d \xi^{2} \ldots d \xi^{d}$. Logo, neste caso, a medida correspondente é

$$
\beta_{\rho}(\xi)=\frac{1}{(4 \pi \rho)^{d / 2}} e^{-\|\xi\|^{2} / 4 \rho} d \xi .
$$


Claramente,

$$
\int_{\mathbb{R}} \beta_{\rho}(\xi) d \xi<\infty
$$

ou seja, $\beta_{\rho}$ é finita. Como $\beta_{\rho}$ é automaticamente positiva, resta então verificarmos a condição (2.2) e que $\beta_{\rho}$ não está concentrada em um conjunto de medida nula de $\mathbb{R}^{d}$. Como $\|\xi\|^{2 \gamma} \exp \left(-\|\xi\|^{2} / 4 \rho\right) \rightarrow 0$, quando $\|\xi\| \rightarrow \infty$, para todo inteiro não negativo $\gamma$, a condição (2.2) vale. Que $\beta_{\rho}$ não está concentrada em um conjunto de medida nula, segue do fato de que $\exp \left(-t^{2} / 4 \rho\right), t \in \mathbb{R}$, é uma função estritamente positiva. 


\section{Capítulo 3}

\section{A Matriz Característica}

Neste capítulo estudaremos a existência de soluções para o Problema 2 descrito no Capítulo 1. Em todo o capítulo, $\mathcal{N}$ e $\mathcal{T}$ são como na Introdução. Note então que $\mathcal{P}$ não tem que ser necessariamente linearmente independente.

Apresentaremos o conceito de matriz característica, matriz esta que depende de $\mathcal{P}$ e $\mathcal{T}$ e que estará inteiramente relacionada com a resolubilidade ou não do problema. Para simplificar o procedimento, assumiremos que os elementos de $\mathcal{P}$ estão ordenados de tal forma que se $\mu<\nu$ então $\alpha_{\mu} \leq \alpha_{\nu}$. Escreveremos então

$$
\operatorname{Grau}(\mathcal{P}):=\left\{\alpha_{\mu}: p_{\mu} \in \mathcal{P}\right\}:=\left\{\gamma_{1}, \gamma_{2}, \ldots, \gamma_{s}\right\}
$$

onde agora $\gamma_{i}<\gamma_{j}$ se $i<j$. Finalmente definiremos

$$
\sigma_{k}:=\text { cardinalidade de }\left\{p_{\mu} \in \mathcal{P}: \alpha_{\mu}=\gamma_{k}\right\}, \quad k=1,2, \ldots, s,
$$

e para cada $k$ em $\{1.2, \ldots, s\}$

$$
\rho_{k}= \begin{cases}0, & \mathrm{k}=1 \\ \sum_{i=1}^{k-1} \sigma_{i} & \text { caso contrário. }\end{cases}
$$


A matriz característica à qual nos referimos acima será obtida seguindo-se os passos abaixo:

Seja $f$ uma função suficientemente diferenciável de $\mathcal{R}_{\mathcal{T}}$ e escrevamos a mesma na forma

$$
f(x)=\sum_{m=1}^{\omega} f_{m}\left(\left\langle a_{m}, x\right\rangle\right),
$$

onde cada $f_{m}$ é real a valores reais. Impondo-se as condições de interpolação obtemos,

$$
p_{\mu}(D) \sum_{m=1}^{\omega} f_{m}\left(\left\langle a_{m}, x_{j}\right\rangle\right)=\Delta\left(x_{j}\right), \quad 1 \leq \mu \leq r, \quad 1 \leq j \leq n .
$$

Como $p(D) f(\langle a, x\rangle)=p(a) f^{(\gamma)}(\langle a, x\rangle)$, qualquer que seja o polinômio de grau $\gamma$, podemos reescrever a expressão acima na forma

$$
\sum_{m=1}^{\omega} p_{\mu}\left(a_{m}\right) f_{m}^{\left(\alpha_{\mu}\right)}\left(\left\langle a_{m}, x_{j}\right\rangle\right)=\Delta\left(x_{j}\right), \quad 1 \leq \mu \leq r, \quad 1 \leq j \leq n .
$$

Lembramos que no sistema acima, as incógnitas são $f_{m}^{\left(\alpha_{\mu}\right)}\left(\left\langle a_{m}, x_{j}\right\rangle\right), \mu=1,2, \ldots, r$, $j=1,2, \ldots, n$. Note que, se para alguma quíntupla $m, \mu_{1}, \mu_{2}, j_{1}, j_{2}$ tivermos, por exemplo, $\alpha_{\mu_{1}}=\alpha_{\mu_{2}}$ e $\left\langle a_{m}, x_{j_{1}}\right\rangle=\left\langle a_{m}, x_{j_{2}}\right\rangle$, então as duas incógnitas $f_{m}^{\left(\alpha_{\mu_{1}}\right)}\left(\left\langle a_{m}, x_{j_{1}}\right\rangle\right)$ e $f_{m}^{\left(\alpha_{\mu_{2}}\right)}\left(\left\langle a_{m}, x_{j_{2}}\right\rangle\right)$ serão iguais. Para evitarmos tais repetições e então podermos resolver o sistema propriamente, vamos introduzir algumas notações adicionais.

Seja $E$ a função que identifica os elementos de $\operatorname{Grau}(\mathcal{P})$. Precisamente, seja $E$ : $\{1,2, \ldots, r\} \rightarrow\{1,2, \ldots, s\}$ a função tal que $E(\mu)=k$ quando, e somente quando $\alpha_{\mu}=\gamma_{k}$. Analogamente, fixado $m=1,2, \ldots, \omega$, escrevamos $\zeta^{m}=\left\{\zeta_{1}^{m}, \zeta_{2}^{m}, \ldots, \zeta_{L_{m}}^{m}\right\}$, para denotar o conjunto de todos os elementos distintos da forma $\left\langle a_{m}, x_{j}\right\rangle$, para algum j. Definamos então $F^{m}:\{1,2, \ldots, n\} \rightarrow\left\{1,2, \ldots, L_{m}\right\}$ de tal modo que $F^{m}(j)=l$ quando, e somente quando, $\left\langle a_{m}, x_{j}\right\rangle=\zeta_{l}^{m}$. Definamos ainda, $\Lambda=\sum_{m=1}^{\omega} L_{m}$.

Escrevendo-se agora

$$
X_{m l}^{k}:=f_{m}^{(\gamma k)}\left(\zeta_{l}^{m}\right), \quad l=1.2, \ldots, L_{m}, \quad m=1,2, \ldots, \omega \cdot \quad k=1,2, \ldots, s,
$$


e

$$
Y:=\left(X_{11}^{1}, X_{12}^{1}, \ldots, X_{1 L_{1}}^{1}, X_{21}^{1}, X_{22}^{1}, \ldots, X_{z L_{\omega}}^{1}, X_{11}^{2}, X_{12}^{2}, \ldots, X_{\omega L_{\omega}}^{s}\right)^{t},
$$

vemos que o sistema (3.1) pode ser escrito na forma

$$
C Y=\left(\Delta\left(x_{1}\right), \Delta\left(x_{2}\right), \ldots, \Delta\left(x_{n}\right)\right)
$$

onde $C$ é a matriz $n r \times s \Lambda$ dada em blocos da seguinte forma: $C$ tem $s$ blocos diagonais $C^{1}, C^{2}, \ldots, C^{s}$, onde cada $C^{k}, k=1,2 \ldots, s$, é uma matriz $n \sigma_{k} \times \Lambda$. Para cada $k=1,2, \ldots, s$, a matriz $C^{k}$ é um bloco dado por

$$
C^{k}=\left(\begin{array}{cccc}
C_{11}^{k} & C_{12}^{k} & \ldots & C_{1 \omega}^{k} \\
C_{21}^{k} & C_{22}^{k} & \ldots & C_{2 \omega}^{k} \\
\vdots & \vdots & \ddots & \vdots \\
C_{\sigma_{k} 1}^{k} & C_{\sigma_{k} 2}^{k} & \ldots & C_{\sigma_{k} \omega}^{k}
\end{array}\right)
$$

onde cada $C_{\eta m}, 1 \leq \eta \leq \sigma_{k}, 1 \leq m \leq \omega$, é uma matriz $n \times L_{m}$ tendo 0 ou $p_{\rho_{k}+\eta}\left(a_{m}\right)$ como entradas. Na verdade, temos que a entrada $j l$ de $C_{\eta m}$ é igual a $p_{\rho_{k}+\eta}\left(a_{m}\right)$ se, e somente, se $F^{m}(j)=l$.

O processo descrito acima parece muito complicado à primeira vista. Apresentamos a seguir dois exemplos para ilustrar tal processo e possivelmente abrandar a confusão gerada pelo excesso de notação. Após os exemplos, voltaremos à nossa discussão.

Exemplo 1: Sejam $d=2, \mathcal{T}=\left\{a_{1}, a_{2}\right\}, \mathcal{N}=\left\{x_{1}, x_{2}\right\}, \mathcal{P}=\left\{p_{1}, p_{2}, p_{3}\right\}$, onde $a_{1}=(-1,2), a_{2}=(1,1), x_{1}=(1,0), x_{2}=(0,1)$, e $\alpha_{1}=\alpha_{2}<a_{3}$. Com esses dados, $\operatorname{Grau}(\mathcal{P})=\left\{\alpha_{1}, \alpha_{3}\right\}, s=2, \sigma_{1}=2$ e $\sigma_{2}=1$. O sistema (3.2) será dado por

$$
\sum_{m=1}^{2} p_{\mu}\left(a_{m}\right) f_{m}^{\alpha_{\mu}}\left(\left\langle a_{m}, x_{j}\right\rangle\right)=\Delta\left(x_{j}\right), \quad j=1,2, \quad \mu=1.2,3 .
$$

Escrevendo-se $\supset\left(x_{j}\right)=\left(b_{i j}, b_{2 j}, b_{3 j}\right), j=1,2$, o sistema acima fica na forma

$$
p_{1}\left(a_{1}\right) f_{1}^{\alpha_{1}}\left(\left\langle a_{1}, x_{1}\right\rangle\right)+p_{1}\left(a_{2}\right) f_{2}^{\alpha_{1}}\left(\left\langle a_{2}, x_{1}\right\rangle\right)=b_{11}
$$




$$
\begin{aligned}
& p_{1}\left(a_{1}\right) f_{1}^{\alpha_{1}}\left(\left\langle a_{1}, x_{2}\right\rangle\right)+p_{1}\left(a_{2}\right) f_{2}^{\alpha_{1}}\left(\left\langle a_{2}, x_{2}\right\rangle\right)=b_{12} \\
& p_{2}\left(a_{1}\right) f_{1}^{\alpha_{2}}\left(\left\langle a_{1}, x_{1}\right\rangle\right)+p_{2}\left(a_{2}\right) f_{2}^{\alpha_{2}}\left(\left\langle a_{2}, x_{1}\right\rangle\right)=b_{21} \\
& p_{2}\left(a_{1}\right) f_{1}^{\alpha_{2}}\left(\left\langle a_{1}, x_{2}\right\rangle\right)+p_{2}\left(a_{2}\right) f_{2}^{\alpha_{2}}\left(\left\langle a_{2}, x_{2}\right\rangle\right)=b_{22} \\
& p_{3}\left(a_{1}\right) f_{1}^{\alpha_{3}}\left(\left\langle a_{1}, x_{1}\right\rangle\right)+p_{3}\left(a_{2}\right) f_{2}^{\alpha_{3}}\left(\left\langle a_{2}, x_{1}\right\rangle\right)=b_{31} \\
& p_{3}\left(a_{1}\right) f_{1}^{\alpha_{3}}\left(\left\langle a_{1}, x_{2}\right\rangle\right)+p_{3}\left(a_{2}\right) f_{2}^{\alpha_{3}}\left(\left\langle a_{2}, x_{2}\right\rangle\right)=b_{32}
\end{aligned}
$$

Efetuando-se os produtos, obtemos

$$
\left\langle a_{1}, x_{1}\right\rangle=-1, \quad\left\langle a_{1}, x_{2}\right\rangle=2, \quad\left\langle a_{2}, x_{1}\right\rangle=\left\langle a_{2}, x_{2}\right\rangle=2 .
$$

Logo, $\zeta^{1}=\left\{\zeta_{1}^{1}, \zeta_{2}^{1}\right\}=\{-1,2\}$ e $\zeta^{2}=\left\{\zeta_{1}^{2}=1\right\}$. Segue que $L_{1}=2, L_{2}=1$, e consequentemente $\Lambda=\sum_{m=1}^{2} L_{m}=3$. Portanto, temos $s \Lambda=2.3=6$ incógnitas para o problema, a saber

$$
\begin{aligned}
& X_{11}^{1}=f_{1}^{\alpha_{1}}\left(\zeta_{1}^{1}\right)=f_{1}^{\alpha_{1}}(-1) \quad X_{12}^{1}=f_{1}^{\alpha_{1}}\left(\zeta_{2}^{1}\right)=f_{1}^{\alpha_{1}}(2) \\
& X_{21}^{1}=f_{2}^{\alpha_{1}}\left(\zeta_{1}^{2}\right)=f_{2}^{\alpha_{1}}(1) \quad X_{11}^{2}=f_{1}^{\alpha_{3}}\left(\zeta_{1}^{1}\right)=f_{1}^{\alpha_{3}}(-1) \\
& X_{12}^{2}=f_{1}^{\alpha_{3}}\left(\zeta_{2}^{1}\right)=f_{1}^{\alpha_{3}}(2) \quad X_{21}^{2}=f_{2}^{\alpha_{3}}\left(\zeta_{1}^{2}\right)=f_{2}^{\alpha_{3}}(1)
\end{aligned}
$$

Os blocos de $C$ são dados por

$$
C^{1}=\left(\begin{array}{ccc}
p_{1}\left(a_{1}\right) & 0 & p_{1}\left(a_{2}\right) \\
0 & p_{1}\left(a_{1}\right) & p_{1}\left(a_{2}\right) \\
p_{2}\left(a_{1}\right) & 0 & p_{2}\left(a_{2}\right) \\
0 & p_{2}\left(a_{1}\right) & p_{2}\left(a_{2}\right)
\end{array}\right)
$$

e

$$
C^{2}=\left(\begin{array}{ccc}
p_{3}\left(a_{1}\right) & 0 & p_{3}\left(a_{2}\right) \\
0 & p_{3}\left(a_{1}\right) & p_{3}\left(a_{2}\right)
\end{array}\right)
$$


e o vetor coluna $Y$ é por

$$
\left(X_{11}^{1}, X_{12}^{1}, X_{21}^{1}, X_{11}^{2}, X_{12}^{2}, X_{21}^{2}\right)^{t}
$$

Exemplo 2: Vamos assumir aqui que $d=3, \mathcal{T}=\left\{a_{1}, a_{2}, a_{3}, a_{4}\right\}, \mathcal{N}=\left\{x_{1}, x_{2}, x_{3}\right\}$ e $\mathcal{P}=\left\{p_{1}, p_{2}\right\}$, onde os elementos dos conjuntos acima serão dados por

$$
\begin{gathered}
a_{1}=(1,0,0), \quad a_{2}=(0,1,0), \quad a_{3}=(0,0,1), \quad a_{4}=(1,1,0), \\
x_{1}=(0,1,0), \quad x_{2}=(0,0,-1), \quad x_{3}=(0,1,-2),
\end{gathered}
$$

e

$$
p_{1}(x)=1, \quad p_{2}(x)=x^{1} .
$$

Nessas condições, $\operatorname{Grau}(\mathcal{P})=\left\{\gamma_{1}, \gamma_{2}\right\}=\{0,1\}, s=2, \sigma_{1}=1$ e $\sigma_{2}=1$. O sistema (3.2) toma a forma

$$
\sum_{m=1}^{4} p_{\mu}\left(a_{m}\right) f_{m}^{\alpha_{\mu}}\left(\left\langle a_{m}, x_{j}\right\rangle\right)=\Delta\left(x_{j}\right) \quad j=1,2,3, \quad \mu=1,2 .
$$

Escrevendo-se $\Delta\left(x_{j}\right)=\left(b_{1 j}, b_{2 j}\right), j=1,2,3$, o sistema acima pode ser escrito na seguinte forma:

$p_{1}\left(a_{1}\right) f_{1}^{\alpha_{1}}\left(\left\langle a_{1}, x_{1}\right\rangle\right)+p_{1}\left(a_{2}\right) f_{2}^{\alpha_{1}}\left(\left\langle a_{2}, x_{1}\right\rangle\right)+p_{1}\left(a_{3}\right) f_{3}^{\alpha_{1}}\left(\left\langle a_{3}, x_{1}\right\rangle\right)+p_{1}\left(a_{4}\right) f_{4}^{\alpha_{1}}\left(\left\langle a_{4}, x_{1}\right\rangle\right)=b_{11}$

$p_{1}\left(a_{1}\right) f_{1}^{\alpha_{1}}\left(\left\langle a_{1}, x_{2}\right\rangle\right)+p_{1}\left(a_{2}\right) f_{2}^{\alpha_{1}}\left(\left\langle a_{2}, x_{2}\right\rangle\right)+p_{1}\left(a_{3}\right) f_{3}^{\alpha_{1}}\left(\left\langle a_{3}, x_{2}\right\rangle\right)+p_{1}\left(a_{4}\right) f_{4}^{\alpha_{1}}\left(\left\langle a_{4}, x_{2}\right\rangle\right)=b_{12}$

$p_{1}\left(a_{1}\right) f_{1}^{\alpha_{1}}\left(\left\langle a_{1}, x_{3}\right\rangle\right)+p_{1}\left(a_{2}\right) f_{2}^{\alpha_{1}}\left(\left\langle a_{2}, x_{3}\right\rangle\right)+p_{1}\left(a_{3}\right) f_{3}^{\alpha_{1}}\left(\left\langle a_{3}, x_{3}\right\rangle\right)+p_{1}\left(a_{4}\right) f_{4}^{\alpha_{1}}\left(\left\langle a_{4}, x_{3}\right\rangle\right)=b_{13}$

$p_{2}\left(a_{1}\right) f_{1}^{\alpha_{2}}\left(\left\langle a_{1}, x_{1}\right\rangle\right)+p_{2}\left(a_{2}\right) f_{2}^{\alpha_{2}}\left(\left\langle a_{2}, x_{1}\right\rangle\right)+p_{2}\left(a_{3}\right) f_{3}^{\alpha_{2}}\left(\left\langle a_{3}, x_{1}\right\rangle\right)+p_{2}\left(a_{4}\right) f_{4}^{\alpha_{2}}\left(\left\langle a_{4}, x_{1}\right\rangle\right)=b_{21}$

$p_{2}\left(a_{1}\right) f_{1}^{\alpha_{2}}\left(\left\langle a_{1}, x_{2}\right\rangle\right)+p_{2}\left(a_{2}\right) f_{2}^{\alpha_{2}}\left(\left\langle a_{2}, x_{2}\right\rangle\right)+p_{2}\left(a_{3}\right) f_{3}^{\alpha_{2}}\left(\left\langle a_{3}, x_{2}\right\rangle\right)+p_{2}\left(a_{4}\right) f_{4}^{\alpha_{2}}\left(\left\langle a_{4}, x_{2}\right\rangle\right)=b_{22}$

$p_{2}\left(a_{1}\right) f_{1}^{\alpha_{2}}\left(\left\langle a_{1}, x_{3}\right\rangle\right)+p_{2}\left(a_{2}\right) f_{2}^{\alpha_{2}}\left(\left\langle a_{2}, x_{3}\right\rangle\right)+p_{2}\left(a_{3}\right) f_{3}^{\alpha_{1}}\left(\left\langle a_{3}, x_{3}\right\rangle\right)+p_{2}\left(a_{4}\right) f_{4}^{\alpha_{1}}\left(\left\langle a_{4}, x_{3}\right\rangle\right)=b_{23}$ 
Efetuando-se os produtos, temos que

$$
\begin{gathered}
\left\langle a_{1}, x_{1}\right\rangle=\left\langle a_{1}, x_{2}\right\rangle=\left\langle a_{1}, x_{3}\right\rangle=\left\langle a_{2}, x_{2}\right\rangle=\left\langle a_{3}, x_{1}\right\rangle=\left\langle a_{4}, x_{2}\right\rangle=0, \\
\left\langle a_{2}, x_{1}\right\rangle=\left\langle a_{2}, x_{3}\right\rangle=\left\langle a_{4}, x_{1}\right\rangle=\left\langle a_{4}, x_{3}\right\rangle=1, \\
\left\langle a_{3}, x_{2}\right\rangle=-1, \quad\left\langle a_{3}, x_{3}\right\rangle=-2 .
\end{gathered}
$$

Logo,

$$
\begin{gathered}
\zeta^{1}=\left\{\zeta_{1}^{1}\right\}=\{0\}, \quad \zeta^{2}=\left\{\zeta_{1}^{2}, \zeta_{2}^{2}\right\}=\{1,0\} \\
\zeta^{3}=\left\{\zeta_{1}^{3}, \zeta_{2}^{3}, \zeta_{3}^{3}\right\}=\{0,-1,-2\}, \quad \zeta^{4}=\left\{\zeta_{1}^{4}, \zeta_{2}^{4}\right\}=\{1,0\} .
\end{gathered}
$$

Segue que $L_{1}=1, L_{2}=2, L_{3}=3$ e $L_{4}=2$ e, consequentemente, $\Lambda=\sum_{m=1}^{4} L_{m}=8$.

Logo, temos $s \Lambda=16$ incógnitas no problema, a saber,

$$
\begin{gathered}
X_{11}^{1}=f_{1}^{\gamma_{1}}\left(\zeta_{1}^{1}\right)=f_{1}^{1}(0), \quad X_{11}^{2}=f_{1}^{\gamma_{2}}\left(\zeta_{1}^{1}\right)=f_{1}^{1}(0), \\
X_{21}^{1}=f_{2}^{\gamma_{1}}\left(\zeta_{1}^{2}\right)=f_{2}^{0}(1), \quad X_{21}^{2}=f_{2}^{\gamma_{2}}\left(\zeta_{1}^{2}\right)=f_{2}^{1}(1), \\
X_{22}^{1}=f_{2}^{\gamma_{1}}\left(\zeta_{2}^{2}\right)=f_{2}^{0}(0), \quad X_{22}^{2}=f_{2}^{\gamma_{2}}\left(\zeta_{2}^{2}\right)=f_{2}^{1}(0), \\
X_{31}^{1}=f_{3}^{\gamma_{1}}\left(\zeta_{1}^{3}\right)=f_{3}^{0}(0), \quad X_{31}^{2}=f_{3}^{\gamma_{2}}\left(\zeta_{1}^{3}\right)=f_{3}^{1}(0), \\
X_{32}^{1}=f_{3}^{\gamma_{1}}\left(\zeta_{2}^{3}\right)=f_{3}^{0}(-1), \quad X_{32}^{2}=f_{3}^{\gamma_{2}}\left(\zeta_{2}^{3}\right)=f_{3}^{1}(-1), \\
X_{33}^{1}=f_{3}^{\gamma_{1}}\left(\zeta_{3}^{3}\right)=f_{3}^{0}(-2), \quad X_{33}^{2}=f_{3}^{\gamma_{2}}\left(\zeta_{3}^{3}\right)=f_{3}^{1}(-2), \\
X_{41}^{1}=f_{4}^{\gamma_{1}}\left(\zeta_{1}^{4}\right)=f_{4}^{0}(1), \quad X_{41}^{2}=f_{4}^{\gamma_{2}}\left(\zeta_{1}^{4}\right)=f_{4}^{1}(1), \\
X_{42}^{1}=f_{4}^{\gamma_{1}}\left(\zeta_{2}^{4}\right)=f_{4}^{0}(0), \quad X_{42}^{2}=f_{4}^{\gamma_{2}}\left(\zeta_{2}^{4}\right)=f_{4}^{1}(0) .
\end{gathered}
$$

Os blocos de $C$ são dados por

$$
C^{1}=\left(\begin{array}{cccccccc}
p_{1}\left(a_{1}\right) & p_{1}\left(a_{2}\right) & 0 & p_{1}\left(a_{3}\right) & 0 & 0 & p_{1}\left(a_{4}\right) & 0 \\
0 & 0 & p_{1}\left(a_{2}\right) & 0 & 0 & p_{1}\left(a_{3}\right) & 0 & p_{1}\left(a_{4}\right) \\
0 & 0 & 0 & 0 & 0 & p_{1}\left(a_{3}\right) & 0 & 0
\end{array}\right)
$$


e

$$
C^{2}=\left(\begin{array}{cccccccc}
p_{2}\left(a_{1}\right) & p_{2}\left(a_{2}\right) & 0 & p_{2}\left(a_{3}\right) & 0 & 0 & p_{2}\left(a_{4}\right) & 0 \\
0 & 0 & p_{2}\left(a_{2}\right) & 0 & 0 & p_{2}\left(a_{3}\right) & 0 & p_{2}\left(a_{4}\right) \\
0 & 0 & 0 & 0 & 0 & p_{2}\left(a_{3}\right) & 0 & 0
\end{array}\right)
$$

e o vetor coluna $Y$ é dado por

$$
Y=\left(X_{11}^{1}, X_{21}^{1}, X_{22}^{1}, X_{31}^{1}, X_{32}^{1}, X_{33}^{1}, X_{41}^{1}, X_{42}^{1}, X_{11}^{2}, X_{21}^{2}, X_{22}^{2}, X_{31}^{2}, X_{32}^{2}, X_{33}^{2}, X_{41}^{2}, X_{42}^{2}\right)^{t}
$$

Podemos agora introduzir o conceito de matriz característica. A matriz $A:=C C^{t}$, onde $C$ é como em (3.2) é denominada matriz característica do Problema 2. Note então que $A$ depende dos dados do problema (mas não da função $f$ ) e portanto a mesma varia de acordo com esses dados. As seguintes propriedades da matriz $A$ são cruciais na prova do teorema principal desta capítulo.

Lema 3.1 i) A matriz característica A é sempre não negativa definida;

ii) A é não singular se, e somente se, a matriz correspondente $C$ tem posto $n r$.

Prova A parte i) segue do fato de que, para todo $c$ em $\mathbb{R}^{n r}$ temos

$$
c^{t} A c=c^{t}\left(C^{t}\right)^{t} C^{t} c=\left(C^{t} c\right)^{t} C^{t} c=\left\|C^{t} c\right\|^{2} \geq 0 .
$$

Passemos então à prova da parte ii). Suponhamos inicialmente que $A$ seja não singular. Por i), ela é então positiva definida (veja [3], página 402) e portanto só temos igualdade em (3.3) quando $c=0$. Vamos usar essa conclusão para mostrar que $C^{t}$ tem nulidade zero. Seja $c$ em $\mathbb{R}^{n r}$ tal que $C^{t} c=0$. Então $c^{t} A c=0$, e pelo que acabamos de concluir. $c=0$. Como $C$ e $C^{t}$ têm a mesma nulidade, segue que $C$ tem nulidade nula. Portanto. seu posto é $n r$.

Reciprocamente, suponhamos que o posto de $C$ é $n r$ e seja $c$ um vetor em $\mathbb{R}^{n r}$ tal que $c^{t} \cdot A c=0$. Pela prova do ítem i), vemos que $C^{t} c=0$. Portanto $c$ está no núcleo

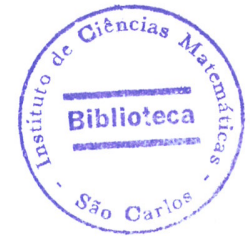


de $C^{t}$. Como $C$ tem posto $n r$, sua nulidade, bem como a de $C^{t}$, é zero. Logo, $c=0$. Portanto $A$ é positiva definida, e consequentemente não singular.

Finalmente podemos discutir a resolubilidade do Problema 2. As notações introduzidas nas discussões acima são utilizadas livremente. Antes porém, enunciaremos o seguinte resultado elementar sobre interpolação hermiteana em uma variável. A prova pode ser encontrada em [6], página 54.

Lema 3.2 Sejam $y_{1}, y_{2}, \ldots y_{n}$ pontos distintos de $\mathbb{R}$. Suponha que para cada $i$ em $\{1,2, \ldots, n\}$ seja dada uma lista de números $\alpha_{i 1}, \alpha_{i 2}, \ldots, \alpha_{i l_{i}}$. Então existe um polinômio $p$ de grau menor ou igual a $n-1+\sum_{i=1}^{n} l_{i}$ tal que $p^{(j)}\left(y_{i}\right)=\alpha_{i j}, 1 \leq j \leq l_{i}$, $1 \leq i \leq n$.

Teorema 3.1 Sejam $\mathcal{N}, \mathcal{P}$ e $\mathcal{T}$ como no Problema 2. Então, existe uma função $f$ em $\mathcal{R}_{\mathcal{T}}$ satisfazendo (1.3), qualquer que seja a função $\Delta$, se, e somente se, o posto de $C^{k}$ for $n \sigma_{k}, k=1,2, \ldots, s$.

Prova Suponhamos inicialmente que o posto de $C^{k}$ seja $n \sigma_{k}, k=1,2, \ldots, s$. Seja $\Delta: \mathcal{N} \rightarrow \mathbb{C}^{r}$ uma função qualquer e escreva

$$
\left(\Delta\left(x_{1}\right), \Delta\left(x_{2}\right), \ldots, \Delta\left(x_{n}\right)\right)=(b(1), b(2), \ldots, b(s))
$$

onde cada vetor $b(k)$ tem $n \sigma_{k}$ componentes. Então, cada sistema linear $C^{k} y(k)=b(k)$ (note que $y(k)$ é então uma variável com $\Lambda$ componentes) tem pelo menos uma solução. Defina agora

$$
\varphi(m, l)= \begin{cases}l, & \mathrm{~m}=1 \\ l+\sum_{i=1}^{m-1} L_{i} & \text { caso contrário. }\end{cases}
$$


Lembrando que $y_{k}^{\varphi(m, l)}$ é a $\varphi(m, l)$ componente do vetor $y_{k}$, para cada valor de $m$ fixado, nós podemos aplicar o lema anterior para obter funções $f_{1}, f_{2}, \ldots, f_{m}$ tais que

$$
f_{m}^{\gamma_{k}}\left(\zeta_{l}^{m}\right)=y_{k}^{\varphi(m, l)}, \quad l=1,2, \ldots, L_{m}, \quad k=1,2, \ldots, s
$$

Como $C$ é diagonal em blocos, vemos então que as conclusões acima implicam que o sistema (3.2) tem solução. Em outras palavras, o Problema 2 tem solução para a $\Delta$ dada.

Reciprocamente, suponha que o Problema 2 tenha solução qualquer que seja a $\Delta$. Então a mesma conclusão vale para o sistema (3.2). Com a mesma notação da parte anterior da prova, e usando-se novamente o fato da matriz $C$ ser diagonal em blocos, vemos que cada sistema $C^{k} y(k)=b(k), k=1,2, \ldots, s$, sempre tem solução, qualquer que seja $b(k)$. Logo, como cada matriz $C^{k}$ é $n \sigma_{k} \times \Lambda$, temos que cada $C^{k}$ tem posto $n \sigma_{k}$.

Finalmente, podemos enunciar e provar o teorema principal desse capítulo. Ele permite-nos concluir a existência ou não de soluções do Problema 2 a partir da singularidade ou não da matriz característica $A$.

Teorema 3.2 Sejam $\mathcal{N}, \mathcal{T}$ e $\mathcal{P}$ como antes. Então, o Problema 2 tem solução qualquer que seja a função $\triangle$ se, e somente se, a matriz característica do problema é nãosingular.

Prova Se o Problema 2 tiver solução qualquer que seja $\Delta$, então, pelo Teorema 3.1. o posto de cada $C^{k}$ é $n \sigma_{k}$. Logo, como $C$ é diagonal em blocos, o posto de $C$ é $n r$. Pelo Lema 3.1-ii), segue que A é não-singular.

Reciprocamente. se Á é não-singular, então o posto de $C$ é $n r$. Consequentemente. o 
posto de cada $C^{k}$ é $n \sigma_{k}$. Daí, pelo Teorema anterior, o problema tem solução qualquer que seja a $\Delta$. 


\section{Capítulo 4}

\section{Algumas Classes de Funções Completamente Monótonas}

Introduziremos neste capítulo, as classes de funções que utilizaremos nas implementações dos problemas estudados nos capítulos anteriores.

Todas as funções que utilizaremos tem, a menos de sinal, derivada completamente monótona. Os Teoremas da Convergência Monótona e Convergência Dominada serão ferramentas básicas no decorrer do capítulo. Tais teoremas podem ser encontrados com demonstrações em [4].

Segundo [9], dizemos que uma função $\phi:(0, \infty) \rightarrow \mathbb{R}$ é completamente monótona em $(0, \infty)$ se a seguinte condição valer:

$$
(-1)^{k} \phi^{(k)}(\xi) \geq 0, \quad \xi>0, \quad k=1,2, \ldots
$$

Se $\theta$ for um inteiro não negativo, escreveremos $C^{\theta}([0, \infty))$. para denotar a família de todas as funções de $[0 . x)$ em $\mathbb{R}$ que possuem derivadas contínuas até ordem 
$\theta$. Derivadas na origem são interpretadas como derivadas unilaterais. Finalmente, escreveremos $\mathcal{C M}_{\theta}$ para denotar o conjunto de todas as funções $\phi:[0, \infty) \rightarrow \mathbb{R}$ satisfazendo:

(i) $\phi \in C^{\theta}([0, \infty))$;

(ii) $\phi$ é completamente monótona em $(0, \infty)$;

(iii) $\phi$ é não constante.

Escreveremos $\mathcal{D} \mathcal{M}_{\theta}$ para denotar o conjunto de todas as funções $\phi:[0, \infty) \rightarrow \mathbb{R}$ satisfazendo:

(i) $\phi \in C^{\theta}([0, \infty))$;

(ii) $\phi^{\prime}$ é completamente monótona em $(0, \infty)$;

(iii) $\phi^{\prime}$ é não constante.

Essas duas classes de funções são relativamente grandes. Alguns exemplos, com $\theta$ arbitrário, são:

1) $\phi(s)=(c+s)^{\delta}, c>0, \delta<0$, é um elemento de $\mathcal{C M}_{\theta}$;

2) $\phi(s)=(c+s)^{\delta}, c>0,0<\delta<1$, é um elemento de $\mathcal{D} \mathcal{M}_{\theta}$;

3) $\phi(s)=\exp (-\rho s), \rho>0$, é um elemento de $\mathcal{C M}_{\theta} ;-\phi$ é um elemento de $\mathcal{D} \mathcal{M}_{\theta}$.

4) $\phi(s)=\ln (s+b) /(s+a), 0<a<b$, é um elemento de $\mathcal{C M}_{\theta}$;

O Teorema de Bernstein [9], mostra que uma função completamente monótona pode ser representada como uma transformada de Laplace de uma medida de Borel positiva e limitada. O enunciado exato desse teorema é:

Lema 4.1 Uma função $\phi$ é completamente monótona em $(0, \infty)$ se, e somente se, ela for uma integral de Riemann-Stieltjes da forma

$$
\phi(s)=\int_{0}^{\infty} e^{-s \xi} d \beta(\xi),
$$

onde $\beta:[0 . x) \rightarrow \mathbb{R}$ é não decrescente e finita. 
Esse teorema permite uma representação análoga para as funções nas duas classes definidas acima. Tal representação é fundamental para a prova dos teoremas nos próximos capítulos.

Teorema 4.1 Uma função $\phi$ pertence à classe $\mathcal{C M}_{\theta}$ se e somente se ela for representável como uma integral da forma

$$
\phi(s)=\int_{0}^{\infty} e^{-s \xi} d \beta(\xi), \quad s \geq 0
$$

onde $\beta$ é não decrescente e finita em $[0, \infty)$ e satisfaz

$$
\int_{0}^{\infty} t^{\theta} d \beta(\xi)<\infty, \quad e \lim _{\epsilon \rightarrow 0^{+}} \int_{\epsilon}^{\infty} d \beta(\xi)>0 .
$$

Prova Primeiramente, suponhamos que $\phi$ tenha a representação integral como no enunciado e mostremos que $\phi \in \mathcal{C M}_{\theta}$. Note que $\phi(s)<\infty, s \in[0, \infty)$, devido à segunda condição em (4.2). Para mostrarmos que $\phi \in \mathcal{C M}_{\theta}$, vamos usar o Lema 2.4. Podemos obviamente assumir que $\theta>0$. Notemos inicialmente que para todo inteiro não negativo $k$,

$$
\frac{d^{k}}{d s^{k}} e^{-s \xi}=(-1)^{k} \xi^{k} e^{-s \xi}, \quad \xi>0, \quad s>0 .
$$

Se $k=\theta$, podemos usar (4.2), para concluir diretamente que

$$
\int_{0}^{\infty}\left|(-1)^{k} \xi^{k} e^{-s \xi}\right| d \beta(\xi) \leq \int_{0}^{\infty} \xi^{\theta}, d \beta(\xi)<\infty .
$$

Se $k<\theta$, então

$$
\begin{aligned}
\int_{0}^{\infty}\left|(-1)^{k} \xi^{k} e^{-s \xi}\right| d \beta(\xi) & \leq\left(\int_{0}^{1}+\int_{1}^{\infty}\right) \xi^{k} d \beta(\xi) \\
& \leq \int_{0}^{1} d \beta(\xi)+\int_{1}^{\infty} \xi^{\theta} d \beta(\xi) \\
& \leq \int_{0}^{\infty} d \beta(\xi)+\int_{0}^{\infty} \xi^{\theta} d \beta(\xi)<x
\end{aligned}
$$


devido a (4.2) e ao fato de $\beta$ ser finita. Pelo Lema 2.4, podemos então concluir que $\phi$ é diferenciável até ordem $\theta$ em $(0, \infty)$ e que

$$
\phi^{(k)}(s)=(-1)^{k} \int_{0}^{\infty} \xi^{k} e^{-s \xi} d \beta(\xi), \quad s>0, \quad k=0,1, \ldots, \theta .
$$

Como a expressão $\xi^{k} \exp (-s \xi)$ define uma função decrescente de $s$, podemos usar o Teorema da Convergência Monótona e obter

$$
\begin{aligned}
\phi^{(k)}(0) & =(-1)^{k} \lim _{s \rightarrow 0^{+}} \int_{0}^{\infty} \xi^{k} e^{-s \xi} d \beta(\xi) \\
& =(-1)^{k} \int_{0}^{\infty} \lim _{s \rightarrow 0^{+}} \xi^{k} e^{-s \xi} d \beta(\xi) \\
& =(-1)^{k} \int_{0}^{\infty} \xi^{k} d \beta(\xi) .
\end{aligned}
$$

Assim, podemos finalmente escrever

$$
\phi^{(k)}(s)=(-1)^{k} \int_{0}^{\infty} \xi^{k} e^{-s \xi} d \beta(\xi), \quad s \geq 0, \quad k=0,1, \ldots, \theta .
$$

Portanto, $\phi \in C^{\theta}([0, \infty))$. O lema anterior garante imediatamente que $\phi$ é completamente monótona. Se $\phi$ fosse constante, então teríamos

$$
0=\phi^{\prime}(0)=\lim _{\epsilon \rightarrow 0^{+}} \int_{\epsilon}^{\infty} \xi d \beta(\xi)>0,
$$

uma contradição. A última desigualdade acima, segue da segunda condição em (4.2), e do fato de $\beta$ ser não decrescente.

Reciprocamente, suponhamos que $\phi \in \mathcal{C M}_{\theta}$. Pelo lema anterior, podemos escrever,

$$
\phi(s)=\int_{0}^{\infty} e^{-s \xi} d \beta(\xi),
$$

onde $\beta$ é não decrescenté. Como $\phi$ é contínua em $s=0$ podemos novamente usar o Teorema da Convergência Monótona e concluir que

$$
\phi(0)=\lim _{s \rightarrow 0^{+}} \phi(s)=\int_{0}^{\infty} d \beta(\xi) .
$$


Portanto, a representação integral de $\phi$ vale para todo $s$. Para mostrarmos que $\beta$ satisfaz (4.2), necessitamos calcular as derivadas de ordem $\leq \theta$ de $\phi$. Faremos os detalhes do cálculo de $\phi^{\prime}$, sendo o cálculo das outras derivadas similares. Se $s>0$ e $h$ é pequeno (e ainda positivo), então

$$
\frac{\phi(s+h)-\phi(s)}{h}=\int_{0}^{\infty} e^{-s \xi}\left(\frac{e^{-h \xi}-1}{h}\right) d \beta(\xi)
$$

e

$$
\left|e^{-s \xi}\left(\frac{e^{-h \xi}-1}{h}\right)\right| \leq e^{-s \xi} \frac{1-e^{-h \xi}}{h} \leq e^{-s \xi} \frac{h \xi}{h}=\xi^{-s \xi} \leq M, \quad \xi \geq 0,
$$

para alguma constante positiva $M$. Como

$$
\int_{0}^{\infty} M d \beta(\xi) \leq M \phi(0)<\infty
$$

podemos usar o Teorema da Convergência Dominada para concluir que

$$
\phi^{\prime}(s)=-\int_{0}^{\infty} e^{-s \xi} \lim _{h \rightarrow 0^{+}}\left(\frac{e^{-h \xi}-1}{h}\right) d \beta(\xi)=\int_{0}^{\infty} t e^{-s \xi} d \beta(\xi) .
$$

Como $\phi$ ' é contínua em $s=0$, o mesmo argumento usado acima implica que a fórmula vale para $s=0$ também. Portanto, após repetirmos procedimento análogo para os outros valores de $k$, podemos concluir que

$$
\phi^{(k)}(s)=(-1)^{k} \int_{0}^{\infty} \xi^{k} e^{-s \xi} d \beta(\xi), \quad s \geq 0, \quad k=0,1, \ldots, \theta .
$$

Assim, a primeira desigualdade em (4.2) segue do fato de $\phi^{(\theta)}(0)<\infty$. Finalmente, se a última desigualdade em (4.2) não valer então

$$
o(s) \leq \int_{0}^{\infty} d \beta(\xi)=0 .
$$

Logo, $\phi$ é constante em $(0, \infty)$. e por continuidade, em $[0, \infty)$. Isto contradiz a condição iii) na definição de $\mathcal{C} \cdot \mathcal{M}_{\theta}$.

Vejamos agora, a versão do teorema anterior, correspondente à classe $\mathcal{D} \mathcal{M}_{\theta}$. 
Teorema 4.2 Uma função $\phi$ pertence á classe $\mathcal{D} \mathcal{M}_{\theta}$ se, e somente se, ela for representável na forma

$$
\phi(s)=\phi(0)+\int_{0}^{\infty} \frac{1-e^{-s \xi}}{\xi} d \beta(\xi), \quad s \geq 0,
$$

onde $\beta$ é não decrescente e finita em $[0, \infty)$, satisfaz

$$
\int_{0}^{\infty} \xi^{\theta-1} d \beta(\xi)<\infty, \quad \lim _{\epsilon \rightarrow 0^{+}} \int_{\epsilon}^{\infty} d \beta(\xi)>0
$$

$e$, ainda,

$$
\int_{\epsilon}^{\infty} \xi^{-1} d \beta(\xi)<\infty, \quad \epsilon>0
$$

Prova A prova é similar à prova do teorema anterior, e portanto alguns detalhes da prova serão omitidos por razões óbvias. Suponhamos que $\phi$ seja representável como no enunciado. A função $\phi$ está bem definida. De fato, a integral faz sentido pois expandindo-se a parte exponencial do integrando em uma série de Mc-Laurin, o termo $\xi$ do denominador é automaticamente cancelado. Ainda, usando (4.5) nós temos que

$$
\int_{0}^{\infty} \frac{1-e^{-s \xi}}{\xi} d \beta(\xi) \leq \int_{0}^{\infty} \frac{1}{\xi} d \beta(\xi)=\lim _{\epsilon \rightarrow 0^{+}} \int_{\epsilon}^{\infty} \frac{1}{\xi} d \beta(\xi)<\infty .
$$

Utilizando-se o Lema 2.4, a primeira condição em (4.4), o Teorema da Convergência Dominada e o Teorema da Convergência Monótona de maneira completamente análoga aquela utilizada no teorema anterior vemos que $\phi$ tem derivadas até ordem $\theta$ em $[0, \infty)$. Além disso as funções $\phi^{(k)}, k=1,2, \ldots, \theta$, são dadas por

$$
\phi^{(k)}(s)=(-1)^{k+1} \int_{0}^{\infty} \xi^{k-1} e^{-s \xi} d \beta(\xi), \quad s \geq 0 .
$$

Assim, $\phi \in C^{\theta}([0, \infty))$. O Lema 4.1 implica diretamente que $\phi^{\prime}$ é completamente monótona em $(0, x)$. Finalmente. $\phi^{\prime}$ é não constante devido à segunda condição em 
(4.4).

Reciprocamente, suponhamos que $\phi \in \mathcal{D M}_{\theta}$. Como $\phi^{\prime}$ é completamente monótona em $(0, \infty)$, segue do Lema 2.4 que

$$
\phi^{\prime}(s)=\int_{0}^{\infty} e^{-s \xi} d \beta(\xi), \quad s>0,
$$

onde $\beta$ é não decrescente em $[0, \infty)$. Afirmamos agora que

$$
\phi(s)=\phi(0)+\int_{0}^{\infty} \phi^{\prime}(\tau) d \tau .
$$

De fato, usando o Teorema Fundamental do Cálculo temos que

$$
\phi(s)=\phi(\epsilon)+\int_{\epsilon}^{s} \phi^{\prime}(\tau) d \tau, \quad \epsilon>0 .
$$

Como $\phi$ e $\phi^{\prime}$ são contínuas em $s=0$, a igualdade desejada segue diretamente da igualdade acima quando $\epsilon \rightarrow 0$. Assim, usando o teorema de Tonelli ([4], pag.184), para trocar a ordem de integração, obtemos

$$
\begin{aligned}
\phi(s) & =\phi(0)+\int_{0}^{s}\left(\int_{0}^{\infty} e^{-\tau \xi} d \beta(\xi)\right) d \tau \\
& =\phi(0)+\int_{0}^{\infty}\left(\int_{0}^{s} e^{-\tau \xi} d \tau\right) d \beta(\xi) \\
& =\phi(0)+\int_{0}^{\infty} \frac{1-e^{-s \xi}}{\xi} d \beta(\xi) .
\end{aligned}
$$

Seguindo-se agora os passos da parte recíproca da prova do teorema anterior podemos concluir que

$$
o^{(k)}(s)=(-1)^{k} \int_{0}^{\infty} \xi^{k-1} e^{-s \xi} d \beta(\xi), \quad s \geq 0, \quad k=1,2, \ldots, \theta .
$$

Portanto, a primeira desigualdade em (4.4) segue do fato de $\phi^{(k)}(0)<x$. A outra desigualdade em (4.4) é obtida como na prova do teorema anterior. Finalmente. para 
mostrarmos que (4.5) vale, observe inicialmente que

$$
1-e^{-\epsilon} \leq 1-e^{-\xi}, \quad \xi \geq \epsilon>0 .
$$

Logo,

$$
\left(1-e^{-\epsilon}\right) \int_{\epsilon}^{\infty} \frac{1}{\xi} d \beta(\xi) \leq \int_{\epsilon}^{\infty} \frac{1-e^{-\xi}}{\xi} d \beta(\xi) \leq \int_{0}^{\infty} \frac{1-e^{-\xi}}{\xi} d \beta(\xi)<\infty .
$$

Como $1-\exp (-\epsilon)>0,(4.5)$ segue imediatamente. Isso conclui a demonstração. 


\section{Capítulo 5}

\section{Soluções do Problema 1 Via Funções Completamente Monótonas}

Neste capítulo, utilizaremos as duas classes de funções introduzidas no capítulo anterior, para construir soluções radiais do Problema 1. Precisamente, o procedimento aqui será o seguinte: fixada uma função $\phi$ em uma das classes de funções do capítulo anterior, mostraremos que existe uma escolha relativamente simples para as funções $h_{1}, h_{2}, \ldots, h_{r}$, escolha essa dependente somente de $\phi$ e $\mathcal{P}$, de modo que é sempre possível encontrar uma função $f$, solução do Problema 1. Quando o conjunto $\mathcal{P}$ for composto por somente um polinômio constante, o procedimento descrito aqui produz soluções para o método das bases de funções radiais explicado no começo da Introdução.

Primeiramente, vejamos o resultado no caso em que utilizamos funções na classe 
$\mathcal{C M}_{\theta}$, para algum $\theta$. Lembramos que $\mathcal{N}$ e $\mathcal{P}$ são antecipadamente dados como nas hipóteses do Problema 1. Além disso definimos aqui, $\gamma$ como na fórmula (2.2).

Teorema 5.1 Seja $\phi$ uma função em $\mathcal{C M}_{2 \gamma}$ e defina $\Phi(x)=\phi\left(\|x\|^{2}\right)$. Se

$$
h_{\nu}:=(-1)^{\alpha_{\nu}} \overline{p_{\nu}}(D) \Phi, \quad \nu=1,2, \ldots, r
$$

então o Problema 1 tem solução. A matriz de interpolação é, neste caso, positiva definida.

Prova Basta mostrarmos a última afirmação do teorema. Para tanto, nós primeiramente utilizamos o Teorema 4.1 para representar $\phi$ na forma

$$
\phi(s)=\int_{0}^{\infty} e^{-s \xi} d \beta(\xi), \quad s \geq 0,
$$

onde $\beta$ satisfaz (4.2) $\operatorname{com} \theta=2 \gamma$. Consequentemente,

$$
\Phi(x)=\int_{0}^{\infty} e^{-\xi\|x\|^{2}} d \beta(\xi), \quad x \in \mathbb{R}^{d} .
$$

Cada bloco $A_{\mu \nu}$ da matriz $A$ do Problema 1 tem entradas $j k$ dadas por

$$
p_{\mu}(D)(-1)^{\alpha_{\nu}} \overline{p_{\nu}}(D) \Phi\left(x_{j}-x_{k}\right)=(-1)^{\alpha_{\nu}} p_{\mu}(D) \overline{p_{\nu}}(D) \Phi\left(x_{j}-x_{k}\right) .
$$

Usando-se o fato de $\int_{0}^{\infty} \xi^{2 \gamma} d \beta(\xi)<\infty$ para verificar as hipóteses do Lema 2.4, e então usando-se o lema propriamente dito, podemos diferenciar sob o sinal de integração de maneira análoga às anteriores. Portanto, as entradas acima podem ser escritas na forma

$$
\int_{0}^{\infty}(-1)^{\alpha_{\nu}} p_{\mu}(D) \overline{p_{\nu}}(D) G_{\xi}\left(x_{j}-x_{k}\right) d \beta(\xi),
$$


onde $G_{\xi}$ é como no Teorema 2.3. Agora, nós simplesmente seguimos os passos do início da prova do Teorema 2.2. Seja $c$ um vetor em $\mathbb{C}^{n r}$ como naquela prova. Então

$$
\bar{c}^{t} A c=\int_{0}^{\infty} \bar{c}^{t} B_{\xi} c d \beta(\xi),
$$

onde $B_{0}$ é a matriz de ordem $n r$ com todas as entradas iguais a 1 e $B_{\xi}, \xi>0$, é a matriz de interpolação correspondente ao Problema 1 quando usamos $h_{\nu}=(-1)^{\alpha_{\nu}} \overline{p_{\nu}}(D) G_{\xi}$. Combinando-se os Teoremas 2.2 e 2.3, vemos que para cada $\xi>0, \bar{c}^{t} B_{\xi} c \geq 0$, com igualdade somente quando $c=0$. Logo, $\bar{c}^{t} A c \geq 0$. Se $c \neq 0$, a continuidade da função $\xi \rightarrow \bar{c}^{t} B_{\xi} c$ em $[0, \infty)$ e a positividade da mesma função em $(0, \infty)$, implicam que $\bar{c}^{t} A c>0$.

Um resultado análogo vale para a classe $\mathcal{D} \mathcal{M}_{\theta}$, mas alguns cuidados extras têm que ser tomados. Na verdade, torna-se mais difícil garantir a não singularidade da matriz A, quando utilizamos funções nesta classe. Hipóteses adicionais fazem-se necessárias. Apresentamos os detalhes em duas versões distintas.

Teorema 5.2 Seja $\phi$ uma função em $\mathcal{D}_{\mathcal{M}_{2 \gamma}}$ e defina $\Phi(x)=-\phi\left(\|x\|^{2}\right)$. Se $\mathcal{P}$ não contém polinômios constantes e

$$
h_{\nu}=(-1)^{\alpha_{\nu}} \overline{p_{\nu}}(D) \Phi, \quad \nu=1,2, \ldots, r
$$

então o Problema 1 tem solução. A matriz de interpolação tem, neste caso, todos os autovalores positivos.

Prova At prova é muito parecida com a prova do teorema anterior. O Teorema 4.2 permite que escrevamos

$$
o(s)=\varphi(0)+\int_{0}^{\infty} \frac{1-e^{-s \xi}}{\xi} d \beta(\xi), \quad s \geq 0 .
$$


onde $\beta$ satisfaz (4.4), com $\theta=2 \gamma$, e (4.5). Logo,

$$
\Phi(x)=-\varphi(0)-\int_{0}^{\infty} \frac{1-e^{-\xi\|x\|^{2}}}{\xi} d \beta(\xi) .
$$

Procedendo-se de maneira análoga àquela do teorema anterior, vemos que cada bloco da matriz $A$ do Problema 1 tem entradas $j k$ dadas por

$$
\int_{0}^{\infty} \xi^{-1}(-1)^{\alpha_{\nu}} p_{\mu}(D) \overline{p_{\nu}}(D) G_{\xi}\left(x_{j}-x_{k}\right) d \beta(\xi) .
$$

Note que a parcela $1 / \xi$ desaparece quando usamos o Lema 2.4, uma vez que ela não depende de $x$ e nenhum dos polinômios é constante. Assim, para $c$ em $\mathbb{C}^{n r}$,

$$
\bar{c}^{t} A c=\int_{0}^{\infty} \xi^{-1} \bar{c}^{t} B_{\xi} c d \beta(\xi),
$$

onde $B_{\xi}$ é como no teorema anterior. O fato de não termos polinômios constantes em $\mathcal{P}$, permite-nos concluir que a função $\xi \rightarrow \xi^{-1} \bar{c}^{t} B_{\xi} c$ está bem definida em 0 . Logo, tal função é contínua em $[0, \infty)$ e, certamente positiva em $(0, \infty)$ devido aos Teoremas 2.2 e 2.3. Assim, o mesmo argumento usado na conclusão da prova do teorema anterior pode ser utilizado aqui, e portanto $A$ é positiva definida.

A hipótese adicional sobre $\mathcal{P}$ pode ser retirada, mas neste caso, alguma informação adicional sobre a função $\phi$ faz-se necessária. A positividade de $\phi$ em 0 é certamente uma das condições que ainda permitem a resolução do Problema 1. Precisaremos da seguinte versão do Teorema de Courant-Fisher ([3]), página 179.

Lema 5.1 Seja A uma matriz hermitiana de ordem $m$. Então o maior autovalor de $A$ é dado por

$$
\lambda_{m}=\max \left\{\frac{\bar{x}^{t} A x}{\bar{x}^{t} x}: x \neq 0\right\} .
$$


O segundo maior autovalor $\lambda_{m-1}$ de A é o valor mínimo da expressão

$$
\max \left\{\frac{\bar{x}^{t} A x}{\bar{x}^{t} x}: x \neq 0, \bar{x}^{t} y=0\right\},
$$

quando y percorre a esfera unitária em $\mathbb{C}^{m}$.

Teorema 5.3 Sejam $\phi$ e $\Phi$ como no teorema anterior. Se $\phi(0)>0$ e as funções $h_{\nu}$ são como no teorema anterior, então o Problema 1 tem solução. A matriz de interpolação, neste caso, é positiva definida ou tem um autovalor negativo e os demais positivos.

Prova Podemos representar $\phi$ como no teorema anterior. Se $\mathcal{P}$ não contém polinômios constantes, a hipótese $\phi(0)>0$ não é necessária, e podemos usar a prova do teorema anterior para concluir que a matriz $A$ é positiva definida. Suponhamos então que $\mathcal{P}$ contenha um polinômio constante. Sem perda de generalidade podemos supor que $p_{1}$ é constante. Aqui, vemos que a entrada $j k$ no bloco $A_{\mu \nu}$ de $A$ é dada por

$$
-\phi(0)-\int_{0}^{\infty} \frac{1-e^{-\xi\left\|x_{i}-x_{j}\right\|^{2}}}{\xi} d \beta(\xi), \quad \mu=\nu=1,
$$

e por

$$
\int_{0}^{\infty} \xi^{-1}(-1)^{\alpha_{\nu}} p_{\mu}(D) \overline{p_{\nu}}(D) G_{\xi}\left(x_{i}-x_{j}\right) d \beta(\xi),
$$

nos demais casos. Para mostrarmos que a matriz $A$ do Problema 1 é não singular, tomemos inicialmente um vetor $c$ em $\mathbb{C}^{n r}$ de tal modo que a soma de suas coordenadas seja 0. Note então que estamos trabalhando com um vetor $c$ pertencente a um subespaço $Z_{n r}$ de $\mathbb{C}^{n r}$ de dimensão $n r-1$. Como no teorema anterior, ainda temos,

$$
\bar{c}^{t} A c=\int_{0}^{\infty} \xi^{-1} \bar{c}^{t} E_{\xi} c d \beta(\xi)>0 .
$$

Esta última informação permite-nos concluir que A tem $n r-1$ autoralores positivos, como passamos a explicar. Seja y um vetor na esfera unitária de $\mathbb{C}^{n r}$. ortogonal a $Z_{n r}$. 
Se $x$ é um vetor não nulo de $\mathbb{C}^{n r}$ e $\bar{x}^{t} y=0$, então certamente $x$ está em $Z_{n r}$. Logo, para qualquer tal $x, \bar{x}^{t} A x>0$ e consequentemente

$$
\max \left\{\frac{\bar{x}^{t} A x}{\bar{x}^{t} x}: x \neq 0, \bar{x}^{t} y=0\right\}>0 .
$$

Portanto, pelo Lema 5.1, o segundo maior autovalor de $-A$ é negativo e assim são todos os demais, menos o maior deles. Equivalentemente, todos os autovalores de $A$, menos o menor deles, são positivos. Resta então mostrar que o último autovalor é negativo. Para tanto tome um vetor $d$ de $\mathbb{C}^{n r}$ com as seguintes características: $d$ tem as primeiras $n$ entradas iguais a 1 e as demais todas nulas. Então, como $\phi(0)>0$,

$$
\bar{d}^{t} A d=\sum_{j=1}^{n} \sum_{k=1}^{n}\left(-\phi(0)-\phi\left(\left\|x_{j}-x_{k}\right\|^{2}\right)\right)<0 .
$$

Assim, pelo Lema 5.1 de novo, concluímos que o maior autovalor de $-A$ é positivo. Portanto o menor autovalor de A é negativo, como queríamos. 


\section{Capítulo 6}

\section{Soluções do Problema 2 Via}

\section{Funções Completamente}

\section{Monótonas}

Neste capítulo, utilizaremos as classes de funções estudadas no Capítulo IV para construir soluções do Problema 2. O processo aqui, é um pouco mais complicado do que aquele desenvolvido no capítulo anterior para obter soluções do Problema 1. Várias notações introduzidas no Capítulo III, são novamente utilizadas, sem qualquer explicação adicional.

Consideraremos aqui a seguinte versão do Problema 2.

Problema 2' Encontrar uma função $f$, única, no espaço gerado pelas nr funções $p_{\mu}(-D) h\left(\cdot-x_{j}\right), \mu=1,2, \ldots, r, j=1,2, \ldots, n$, onde $h$ é uma função em $\mathcal{R}_{\mathcal{T}}$, de modo que $f$ satisfaça (1.3), qualquer que seja $\Delta$. 
Procedendo-se como na discussão do Problema 1, vemos que, fixada a função $h$, o problema acima tem solução se, e somente se, a seguinte matriz

$$
\left(p_{\mu}(D) p_{\nu}(-D) h\left(x_{j}-x_{k}\right)\right)_{\mu, \nu=1 ; j, k=1}^{r ; n}
$$

for não singular. Como esperado, a não singularidade da matriz acima, estará diretamente ligada à não singularidade da matriz característica do problema.

Inicialmente, apresentamos o seguinte resultado técnico referente ao conceito de matriz característica introduzido no Capítulo III. Ele permitirá que façamos a conexão acima mencionada. Para qualquer $a$ em $\mathcal{T}$, escreveremos $\langle a, \mathcal{N}\rangle$ para denotar o conjunto $\{\langle a, x\rangle: x \in \mathcal{N}\}$.

Lema 6.1 Fixados $\mathcal{N}, \mathcal{T}$ e $\mathcal{P}$, considere a matriz característica $A=C C^{t}$ do Problema 2 correspondente. Para cada $k$ em $\{1,2, \ldots, s\}$, seja $A^{k}$ o bloco correspondente ao produto efetuado com o bloco $C^{k}$ de $C$ por $\left(C^{k}\right)^{t}$. Se c é um vetor de $\mathbb{R}^{n \sigma_{k}}$ com coordenadas $c_{\nu j}, 1 \leq \nu \leq \sigma_{k}, 1 \leq j \leq n$, então

$$
c^{t} A^{k} c=\sum_{m=1}^{M} \sum_{\lambda \in\left\langle a_{m}, \mathcal{N}\right\rangle}\left(\sum_{\mu=1}^{\sigma_{k}} p_{\rho_{k}+\mu}\left(a_{m}\right) \sum_{\left\{i:\left\langle a_{m}, x_{i}\right\rangle=\lambda\right\}} c_{\mu i}\right)^{2} .
$$

Prova Levando-se em conta a descrição dos elementos de $C^{k}$ dada no Capítulo III, temos que a entrada $i j$ no bloco $\mu \nu$ de $A^{k}$ será dada por

$$
A_{\mu \nu i j}^{k}:=\sum_{m=1}^{\omega} \sum_{l=1}^{L_{m}}\left(C_{\mu m}^{k}\right)_{i l}\left(C_{\nu n}^{k}\right)_{j l}, \quad \mu, \nu=1,2, \ldots, \sigma_{k}, \quad i, j=1,2, \ldots, n .
$$

Por aquela mesma descrição, vemos que as contribuições não nulas nessa soma ocorrem somente quando $F^{m}(i)=l$ e $F^{m}(j)=l$. Quando este for o caso, o somando 
correspondente é $p_{\rho_{k}+\mu}\left(a_{m}\right) p_{\rho_{k}+\mu}\left(a_{m}\right)$. Lembrando agora a definição de $F^{m}$, temos que $F^{m}(i)=F^{m}(j)=l$ se, e somente se, $\left\langle a_{m}, x_{i}\right\rangle=\zeta_{l}^{m}=\left\langle a_{m}, x_{j}\right\rangle$. Portanto,

$$
A_{\mu \nu i j}^{k}=\sum_{\left\{m:\left\langle a_{m}, x_{i}\right\rangle=\left\langle a_{m}, x_{j}\right\rangle\right\}} p_{\rho_{k}+\mu}\left(a_{m}\right) p_{\rho_{k}+\nu}\left(a_{m}\right) .
$$

Finalmente, usando a igualdade acima, obtemos

$$
\begin{aligned}
c^{t} A^{k} c & =\sum_{\mu, \nu=1}^{\sigma_{k}} \sum_{i, j=1}^{n} c_{\mu i} c_{\nu j} \sum_{\left\{m:\left\langle a_{m}, x_{i}\right\rangle=\left\langle a_{m}, x_{j}\right\rangle\right\}} p_{\rho_{k}+\mu}\left(a_{m}\right) p_{\rho_{k}+\nu}\left(a_{m}\right) \\
& =\sum_{m=1}^{\omega} \sum_{\mu, \nu=1}^{\sigma_{k}} \sum_{\lambda \in\left\langle a_{m}, \mathcal{N}\right\rangle\left\langle i, j:\left\langle a_{m}, x_{i}\right\rangle=\left\langle a_{m}, x_{j}\right\rangle=\lambda\right\}} c_{\mu i} c_{\nu j} p_{\rho_{k}+\mu}\left(a_{m}\right) p_{\rho_{k}+\nu}\left(a_{m}\right) .
\end{aligned}
$$

A última soma acima é simétrica em $i$ e $j$, e, $\mu$ e $\nu$. Logo,

$$
c^{t} A^{k} c=\sum_{m=1}^{\omega} \sum_{\lambda \in\left\langle a_{m}, \mathcal{N}\right\rangle}\left(\sum_{\mu=1}^{\sigma_{k}} p_{\rho_{k}+\mu}\left(a_{m}\right) \sum_{\left\{i:\left\langle a_{m}, x_{i}\right\rangle=\lambda\right\}} c_{\mu i}\right)^{2},
$$

completando a prova do teorema.

Já podemos enunciar e provar os resultados principais desse capítulo. Denotaremos por $\Xi$ o maior elemento do conjunto $\operatorname{Grau}(\mathcal{P})$.

Teorema 6.1 Sejam $\mathcal{N}, \mathcal{T}$ e $\mathcal{P}$ como no Problema 2'. Sejam $\phi_{1}, \phi_{2}, \ldots, \phi_{\omega}$ funções em $\mathcal{C M}_{2 \Xi}$ e defina a seguinte função $\Phi$ em $\mathcal{R}_{\mathcal{T}}$ :

$$
\Phi(x):=\sum_{m=1}^{\omega} \phi_{m}\left(\left\langle a_{m}, x\right\rangle^{2}\right) .
$$

Então a matriz

$$
B=\left(p_{\mu}(D) p_{\nu}(-D) \Phi\left(x_{j}-x_{k}\right)\right)_{\mu, \nu=1 ; j, k=1}^{r ; n}
$$

é não negativa definida. 
Prova Pelo Teorema 4.1 podemos escrever cada $\phi_{m}$ na forma

$$
\phi_{m}(s)=\int_{0}^{\infty} e^{-s \xi} d \beta_{m}(\xi), \quad s \geq 0
$$

onde $\beta_{m}$ é uma medida de Borel positiva e finita em $[0, \infty)$, satisfazendo as condições (4.2). Logo,

$$
\phi_{m}\left(\left\langle a_{m}, x\right\rangle^{2}\right)=\int_{0}^{\infty} e^{-\xi\left\langle-a_{m}, x\right\rangle^{2}} d \beta_{m}(\xi) .
$$

Usando a mesma representação integral utilizada no começo da prova do Teorema 2.3, podemos escrever

$$
\phi_{m}\left(\left\langle a_{m}, x\right\rangle^{2}\right)=\frac{1}{2 \sqrt{\pi}} \int_{0}^{\infty} \int_{\mathbb{R}} e^{i \sqrt{\xi}\left\langle a_{m}, x\right\rangle w} e^{-w^{2} / 4} d w d \beta_{m}(\xi) .
$$

Procedendo-se como no Capítulo II, vemos que

$$
\begin{aligned}
& p_{\mu}(D) p_{\nu}(-D) \phi_{m}\left(\left\langle a_{m}, x\right\rangle^{2}\right)= \\
& \quad \frac{1}{2 \sqrt{\pi}} \int_{0}^{\infty} \int_{\mathbb{R}} p_{\mu}\left(i \sqrt{\xi} w a_{m}\right) p_{\nu}\left(i \sqrt{\xi} w a_{m}\right) e^{i \sqrt{\xi}\left\langle a_{m}, x\right\rangle w} e^{-w^{2} / 4} d w d \beta_{m}(\xi) .
\end{aligned}
$$

Usando agora a homogeneidade dos polinômios de $\mathcal{P}$, podemos escrever

$$
\begin{aligned}
& p_{\mu}(D) p_{\nu}(-D) \phi_{m}\left(\left\langle a_{m}, x_{j}-x_{k}\right\rangle^{2}\right)= \\
& \quad \frac{1}{2 \sqrt{\pi}} \int_{0}^{\infty} \int_{\mathbb{R}}(i \sqrt{\xi} w)^{\alpha_{\mu}}(-i \sqrt{\xi} w)^{\alpha_{\nu}} p_{\mu}\left(a_{m}\right) p_{\nu}\left(a_{m}\right) e^{i \sqrt{\xi}\left\langle a_{m}, x_{j}-x_{k}\right\rangle w} \times \\
& \quad \times e^{-w^{2} / 4} d w d \beta_{m}(\xi) .
\end{aligned}
$$

Usando então a definição de $\Phi$ e a última igualdade, temos que, para qualquer $c$ em $\mathbb{R}^{n r}$.

$$
\begin{aligned}
c^{t} B c= & \frac{1}{2 \sqrt{\pi}} \sum_{m=1}^{\omega} \int_{0}^{\infty} \int_{\mathbb{R}} \sum_{\mu, \nu=1}^{r} \sum_{j, k=1}^{n} c_{\mu j} c_{\nu k}(i \sqrt{\xi} w)^{\alpha_{\mu}}(-i \sqrt{\xi} w)^{\alpha_{\nu}} \times \\
& \times p_{\mu}\left(a_{m}\right) p_{\nu}\left(a_{m}\right) e^{i \sqrt{\xi}\left\langle a_{m}, x_{j}-x_{k}\right\rangle} e^{-w^{2} / 4} d u d 3_{m}(\xi) .
\end{aligned}
$$


Reagrupando-se e reordenando-se as somas, podemos finalmente escrever

$$
\begin{aligned}
c^{t} B c= & \frac{1}{2 \sqrt{\pi}} \sum_{m=1}^{\omega} \int_{0}^{\infty} \int_{\mathbb{R}}\left|\sum_{\mu=1}^{r}\left[(i \sqrt{\xi} w)^{\alpha_{\mu}} p_{\mu}\left(a_{m}\right) \sum_{j=1}^{n} c_{\mu j} e^{i \sqrt{\xi}\left\langle a_{m}, x_{j}\right\rangle w}\right]\right|^{2} e^{-\frac{w^{2}}{4}} d w d \beta_{m}(\xi) \\
= & \frac{1}{2 \sqrt{\pi}} \sum_{m=1}^{\omega} \int_{0}^{\infty} \int_{\mathbb{R}} \mid \sum_{\gamma \in \operatorname{Grau}(\mathcal{P})} \sum_{\lambda \in\left\langle a_{m}, \mathcal{N}\right\rangle}\left[\sum_{\left\{\mu: \alpha_{\mu}=\gamma\right\}} p_{\mu}\left(a_{m}\right) \times\right. \\
& \left.\times \sum_{\left\{\nu: a_{m} x_{\nu}=\lambda\right\}} c_{\mu \nu}\right]\left.(i \sqrt{\xi} w)^{\gamma} e^{i \sqrt{\xi} \lambda w}\right|^{2} e^{-w^{2} / 4} d w d \beta_{m}(\xi) .
\end{aligned}
$$

Como a útima expressão acima é obviamente não negativa, a prova do teorema está completa.

Teorema 6.2 Nas condições do Teorema 6.1, temos que a matriz B lá descrita é positiva definida se, e somente se, a matriz característica $A$ do problema for positiva definida.

Prova Suponhamos inicialmente que a matriz $B$ não seja positiva definida. Pelo teorema anterior, existe então $c \in \mathbb{R}^{n r}, c \neq 0$, tal que $c^{t} B c=0$. Olhando-se para o cálculo de $c^{t} B c$ feito na prova do teorema anterior e lembrando que cada $\beta_{m}$ não está concentrada em um conjunto de medida nula, concluímos que cada função $V_{m, \xi}$ : $\mathbb{R} \rightarrow \mathbb{R}$ dada por

$$
V_{m, \xi}(w)=\sum_{\gamma \in \operatorname{Grau}(\mathcal{P})} \sum_{\lambda \in\left\langle a_{m}, \mathcal{N}\right\rangle}\left[\sum_{\left\{\mu: \alpha_{\mu}=\gamma\right\}} p_{\mu}\left(a_{m}\right) \cdot \sum_{\left\{j:\left\langle a_{m}, x_{j}\right\rangle=\lambda\right\}} c_{\mu j}\right](i \sqrt{\xi} w)^{\gamma} e^{i \sqrt{\xi} \lambda w},
$$

deve anular-se, qualquer que seja $\xi>0$. Note agora que cada $I_{m . \xi}^{-}$é uma combinação linear de funções do tipo estudado no Lema 2.2. Além disso os coeficientes nas combinações lineares acima não dependem de $\xi$. Aplicando então o Corolário 2.1 para um valor de $\xi$ fixado, concluímos que

$$
\sum_{\left\{\mu: \alpha_{\mu}=\gamma\right\}} p_{\mu}\left(a_{m}\right) \sum_{\left\{j:\left\{a_{m}, x_{j}=\lambda\right\}\right.} c_{\mu j}=0
$$


qualquer que seja $m \in\{1,2, \ldots, \omega\}, \gamma \in \operatorname{Grau}(\mathcal{P})$ e $\lambda \in\left\langle a_{m}, \mathcal{N}\right\rangle$. Pelo Lema 6.1, a matriz característica $A$ do problema satisfaz $c^{t} \cdot A^{k} c=0$. Como $A$ é não negativa definida e $c \neq 0, A$ é singular.

Reciprocamente, suponhamos que $A$ não seja positiva definida. Utilizando-se a expressão no final da prova do Lema 6.1 , vemos que existe então $c \in \mathbb{R}^{n r}, c \neq 0 \mathrm{e}$ $m \in\{1,2, \ldots, \omega\}$ de modo que para todo $\lambda \in\left\langle a_{m}, \mathcal{N}\right\rangle$,

$$
\sum_{\left\{\mu: \alpha_{\mu}=\gamma\right\}} p_{\mu}\left(a_{m}\right) \sum_{\left\{j:\left\{a_{m}, x_{j}\right\rangle=\lambda\right\}} c_{\mu j}=0 .
$$

Consequentemente, as funções $V_{m, \xi}$ da parte anterior são todas nulas. Em particular, $c^{t} B c=0$. Assim, $B$ não é positiva definida.

Quando utilizamos funções na classe $\mathcal{D} \mathcal{M}_{2 \Xi}$, os resultados obtidos só equiparam-se ao do teorema anterior, quando $\mathcal{P}$ não contém polinômios constantes.

Teorema 6.3 Sejam $\mathcal{N}, \mathcal{T}$ e $\mathcal{P}$ como no problema 2'. Sejam $\phi_{1}, \phi_{2}, \ldots, \phi_{\omega}$ funções em $\mathcal{D M}_{2}$ e defina $\Phi$ em $\mathcal{R}_{\mathcal{T}}$ como no Teorema 6.1. Se $\mathcal{P}$ não contém polinômios contantes então a matriz característica do problema é positiva definida se, e somente se a matriz B, definida como no Teorema 6.1, é não singular.

Prova Usando o Teorema 4.2, podemos representar cada $\phi_{m}$ na forma

$$
\phi_{m}(s)=\int_{0}^{\infty} \frac{1-e^{-\xi s}}{\xi} d \beta_{m}(\xi)
$$

onde $\beta_{m}$ é uma medida de Borel positiva em $[0, \infty)$ satisfazendo as condições (4.4) e (4.j) $(\operatorname{com} \theta=2 \Xi)$. Então, podemos escrever

$$
p_{\mu}(D) p_{\nu}(-D) \phi_{m}\left(\left\langle a_{m}, x\right\rangle^{2}\right)=-\int_{0}^{\infty} \xi^{-1} p_{\mu}(D) p_{\nu}(-D) e^{-\left\langle a_{m}, x\right\rangle^{2} \xi} d \beta_{m}(\xi) .
$$


Seguindo-se os passos da prova do Teorema 6.1, se $c$ é um vetor em $\mathbb{R}^{n r}$ temos que

$$
\begin{aligned}
c^{t} B c=- & \left.\frac{1}{2 \sqrt{\pi}} \sum_{m=1}^{\omega} \int_{0}^{\infty} \int_{\mathbb{R}}\right|_{\gamma \in \operatorname{Grau}(\mathcal{P})} \sum_{\left\{\mu: \alpha_{\mu}=\gamma\right\}} p_{\mu}\left(a_{m}\right) \times \\
& \left.\sum_{\lambda \in\left\langle a_{m}, \mathcal{N}\right\rangle} \sum_{\left\{j:\left\langle a_{m}, x_{j}\right\rangle=\lambda\right\}} c_{\mu j}(i \sqrt{\xi} w)^{\gamma} e^{i \sqrt{\xi} \lambda w}\right|^{2} \xi^{-1} e^{-w^{2} / 4} d w d \beta_{m}(\xi) .
\end{aligned}
$$

Logo, se a matriz característica do problema é positiva definida, parte da prova do teorema anterior pode ser utilizada para concluirmos que $-B$ é positiva definida e vice e versa.

No caso geral, temos o seguinte resultado.

Teorema 6.4 Assuma as hipóteses do teorema anterior. Se $\phi_{m}(0)>0$, para algum $m$ e a matriz característica do problema é positiva definida, então a matriz $B$ é não singular.

Prova Assuma as hipóteses do teorema. Se $\mathcal{P}$ não contém polinômios constantes, então o resultado segue do teorema anterior, utilizando-se somente o fato de $A$ ser positiva definida. Assuma então que $\mathcal{P}$ contém polinômios contantes. Obviamente, só precisamos analisar o caso em que $\mathcal{P}$ contém um único tal polinômio. Sem perda de generalidade, vamos assumir então que $p_{1}=1$ e que os demais polinômios de $\mathcal{P}$ têm grau no mínimo 1. Com a notação do teorema anterior, temos que

$$
p_{1}(D) p_{1}(-D) \phi_{m}\left(\left\langle a_{m}, x\right\rangle^{2}\right)=\int_{0}^{\infty} \frac{1-e^{-\left\langle a_{m}, x\right\rangle^{2} \xi}}{\xi} d \beta_{m}(\xi) .
$$

Para mostrarmos que a matriz $B$ é não singular, faremos como no Teorema 5.3. Seja $c \in \mathbb{R}^{n r}$ um vetor cuja soma das coordenadas seja zero. Logo $c$ pertence a um subespaço $Z_{n r}$ de $\mathbb{R}^{n r}$ de dimensão $n r-1$. Para tal $c$,

$$
\sum_{j, k=1}^{n} c_{1 j} c_{1 k} \int_{0}^{\infty} \frac{1-e^{\left\langle a_{m}, x\right\rangle^{2} \xi}}{\xi} d \beta_{m}(\xi)=-\sum_{j, k=1}^{n} c_{1 j} c_{1 k} \int_{0}^{\infty} \frac{e^{-\left\langle a_{m}, x\right\rangle^{2} \xi}}{\xi} d \beta_{m}(\xi) .
$$


Pela prova do teorema anterior, concluímos então que $c^{t} B c<0$, para $c$ em $Z_{n r}$. Como na prova do Teorema 5.3, temos então que $B$ possui $n r-1$ autovalores negativos. Para mostrar que o último autovalor é positivo, tomemos $d$ em $\mathbb{R}^{n r}$ de tal modo que suas $j$ primeiras entradas sejam iguais a 1 e as demais nulas. Como $\phi_{m}$ é não decrescente, temos que

$$
d^{t} B d=\sum_{m=1}^{\omega} \sum_{j, k=1}^{n} \phi_{m}\left(\left\langle a_{m},\left(x_{j}-x_{k}\right)\right\rangle^{2}\right) \geq n^{2} \sum_{m=1}^{\omega} \phi_{m}(0)>0 .
$$

Assim, pelo Lema 5.1, o maior autovalor de $B$ é positivo. Portanto, $B$ é não singular, concluindo a prova. 


\section{Bibliografia}

[1] Akhiezer, N. I., Lectures on Integral Transforms, Translations of Mathematical Monographs, Vol. 70, Amer. Math. Soc., Providence, 1988.

[2] Braess, D. e A. Pinkus, Interpolation by Ridge Functions, J. Approx. Theory, 73 (1993), 218-236.

[3] Horn, R.A. e C. R. Johnson, Matrix Analysis, Cambridge University Press, Cambridge, 1985.

[4] Jones, F., Lebesgue Integration on Euclidean Space, Jones and Bartlett Publishers, Boston, 1993.

[5] Levesley, J. e X. Sun, Scattered Hermite Interpolation by Ridge Functions, Numer. Funct. Anal. Optim., 16:7,8 (1995), 989-1001.

[6] Powel, M. J. D., Approximation Theory and Methods, Cambridge University Press, Cambridge, 1981.

[7] Sun, X., Scattered Hermite Interpolation Using Radial Basis Functions, Linear Algebra and its Appl., 207 (1994), 135-146.

[8] Weinmannn, A.. The Interpolation Problem for Ridge Functions, Numer. Func. Anal. Optim., 15:1,2 (1994), 183-186. 
[9] Widder, D., V., An Introduction to Transform Theory, Academic Press, New York, 1971. 